\title{
Multiscale analysis of fluxes at the turbulent/non-turbulent interface in high Reynolds number boundary layers
}

\author{
Jimmy Philip, Charles Meneveau, ${ }^{\text {a) }}$ Charitha M. de Silva, and Ivan Marusic
}

Department of Mechanical Engineering, University of Melbourne, VIC 3010, Australia

(Received 26 September 2013; accepted 17 December 2013;

published online 13 January 2014)

\begin{abstract}
Analysis of fluxes across the turbulent/non-turbulent interface (TNTI) of turbulent boundary layers is performed using data from two-dimensional particle image velocimetry (PIV) obtained at high Reynolds numbers. The interface is identified with an iso-surface of kinetic energy, and the rate of change of total kinetic energy $(K)$ inside a control volume with the TNTI as a bounding surface is investigated. Features of the growth of the turbulent region into the non-turbulent region by molecular diffusion of $K$, viscous nibbling, are examined in detail, focussing on correlations between interface orientation, viscous stress tensor elements, and local fluid velocity. At the level of the ensemble (Reynolds) averaged Navier-Stokes equations (RANS), the total kinetic energy $K$ is shown to evolve predominantly due to the turbulent advective fluxes occurring through an average surface which differs considerably from the local, corrugated, sharp interface. The analysis is generalized to a hierarchy of length-scales by spatial filtering of the data as used commonly in Large-Eddy-Simulation (LES) analysis. For the same overall entrainment rate of total kinetic energy, the theoretical analysis shows that the sum of resolved viscous and subgrid-scale advective flux must be independent of scale. Within the experimental limitations of the PIV data, the results agree with these trends, namely that as the filter scale increases, the viscous resolved fluxes decrease while the subgrid-scale advective fluxes increase and tend towards the RANS values at large filter sizes. However, a definitive conclusion can only be made with fully resolved three-dimensional data, over and beyond the large dynamic spatial range presented here. The qualitative trends from the measurement results provide evidence that large-scale transport due to the energy-containing eddies determines the overall rate of entrainment, while viscous effects at the smallest scales provide the physical mechanism ultimately responsible for entrainment. Data spanning over a decade in Reynolds number suggest that the fluxes (or the entrainment velocity) scale with the friction velocity (or equivalently the local turbulent fluctuating velocity), whereas Taylor microscale and boundary-layer thickness are the appropriate length scales at small and large filter sizes, respectively. (C) 2014 AIP Publishing LLC. [http://dx.doi.org/10.1063/1.4861066]
\end{abstract}

\section{INTRODUCTION}

Physical processes occurring at the sharp and corrugated surface separating turbulent from nonturbulent regions in shear flows such as jets and boundary layers have elicited considerable interest for over half a century. ${ }^{1-6}$ Such surfaces are subjected to spatially varying advection and deformation by turbulent eddies at various scales that tend to increase the surface area. Conversely, molecular processes that typically occur at small diffusive scales in the immediate vicinity of the surface tend to smooth, and thus reduce, the surface area. Work in the 1980s and 1990s had focussed on a possibly fractal structure of surfaces in turbulence (cloud boundaries, flames, interfaces) as a reflection of deformations caused by a hierarchy of eddy sizes in the turbulent (Kolmogorov's) inertial range. ${ }^{7-11}$

\footnotetext{
a) Permanent address: Department of Mechanical Engineering and Center for Environmental and Applied Fluid Mechanics, The Johns Hopkins University, 3400 N. Charles Street, Baltimore, MD 21218, USA.
} 
More recently, there has been growing interest in the structure of turbulence and transport in the vicinity of the turbulent/non-turbulent interface (TNTI hereafter). ${ }^{6}{ }^{12-14}$ The recent work has focussed mostly on turbulent jets at moderate Reynolds numbers, specifically on the question of whether the entrainment of the turbulent region into the non-turbulent region is dominated by large-scale processes through the energy-containing eddies (engulfment), or whether it is due to small-scale diffusive processes occurring at the interface (viscous nibbling). Recent papers ${ }^{6,13,15,16}$ provide evidence supporting both views. (As an aside, we note that the term "engulfment" is typically defined in qualitative terms in the literature. If engulfment is used in the sense of ingestion of nonturbulent fluid by the surrounding turbulent fluid, ${ }^{17}$ then it has been observed that the contribution to total entrainment by the mass associated with the "ingestion" of fluid is small. ${ }^{6,12}$ In such a definition, it is difficult to separate particular length-scales responsible for the phenomena and yet, relating large and small scales to engulfment and nibbling, respectively, is not uncommon. ${ }^{13,18}$ Here we propose to associate the concept of "engulfment" to any non-viscous transport mechanism that can be associated to the large-scale energy containing eddies. ${ }^{16}$ Then a precise definition can be proposed.) The question of engulfment versus nibbling has thus far not been settled, but as mentioned by Mathew and Basu, ${ }^{12}$ if the interface has fractal scaling (outlined by Sreenivasan, Ramshankar, and Meneveau ${ }^{10}$ and Meneveau and Sreenivasan ${ }^{11}$ ), the two views can be reconciled: the engulfment caused by inertial flow structures at large and intermediate sizes increases the surface area where diffusive processes can then act more effectively over larger areas but at the small scales. A recent analysis by de Silva et al. ${ }^{19}$ has provided further evidence of fractal scaling of the TNTI using high Reynolds number boundary layer data sets. However, a purely geometric analysis of surface area is, by itself, insufficient to answer questions directly related to physical fluxes and rates of entrainment.

The aim of the present work is to examine transport processes along the TNTI at various scales, to further clarify what physical mechanisms are responsible at each scale and to shed new light upon the long-standing questions about large-scale engulfment versus small-scale viscous nibbling. We shall consider the growth of the turbulent region via the fluxes of kinetic energy across the TNTI. The fluxes separate into two categories: the viscous flux (at small scales) which we associate with "nibbling", and the advective flux (at large scales) to be associated with "engulfment". Taking our cue from the fractal description of the interface, we intend to ascertain/understand the scenario that at the smallest scales only viscous flux is present, whereas at the largest scales only advective flux is present, while at in-between scales both operate; however, irrespective of the scale the total flux is the same. Furthermore, we aim to examine the physical processes by which the viscous and advective fluxes transfer kinetic energy across the interface, and how they change, if at all, with length scale.

In order to enable a clear separation of scales, flows at very high Reynolds numbers are ideally required, with at least a factor $\delta / \eta \sim 10^{3}$ available (where $\delta$ is a large-scale such as the boundary layer thickness and $\eta$ a small scale such as the Kolmogorov scale), in order to account for a factor 10 on each side of the largest and smallest scales. Also the turbulent velocity field needs to be measured with sufficient accuracy to apply criteria that can discern the TNTI based on dynamically relevant variables instead of relying only on properties of surrogate, non-dynamic fields such as passive scalars. The flow has to be probed in more than one dimension, ideally in three dimensions, but planar cuts rather than point-wise measurements can already provide meaningful results. Furthermore, for statistical convergence one requires large amounts of data acquired under well-controlled experimental conditions. Over the past decade, significant progress has been made in experimental techniques such as high-resolution Particle Image Velocimetry (PIV). For example, planar PIV data by Westerweel et al. ${ }^{13}$ Westerweel et al. ${ }^{6}$ and Khashehchi et al. ${ }^{20}$ have been used to study the conditional statistics at the TNTI in axisymmetric jets at moderate $R e=2000$ to 6500 (based on the jet diameter and nozzle velocity). They have found, as first hypothesised by Corrsin and Kistler $^{1}$ (also see Ref. 21) that there exists a "jump" in the velocity tangential to the TNTI, which is related to the jump in the Reynolds stress gradient. In fact, such a jump was also found by Chen and Blackwelder ${ }^{22}$ from the conditional profiles using hot-wires, even though it was called a "shear layer". Furthermore, Westerweel et al. ${ }^{6}$ have used $R e=2000$ jets to measure viscous vorticity fluxes near the TNTI for understanding the nibbling mechanism. Direct Numerical Simulations (DNSs) by da Silva, dos Reis, and Pereira ${ }^{18}$ and da Silva and Pereira ${ }^{23}$ (of planar jets) have confirmed many of the conclusions regarding conditional averages and have also found that the size of the 
jump- or shear-layer thickness is related to the vortices residing on the TNTI. For boundary layers too, Semin et $a .^{24}$ (at $R e_{\tau}=600$ ) and Chauhan et al. ${ }^{25}$ (at $R e_{\tau}=14500$ ) have found that the conditional profiles satisfy Reynolds' jump conditions. ${ }^{21}$

To elucidate the role of large- and small-scale mechanisms of entrainment, Philip and Marusic ${ }^{16}$ pointed out that it is informative to separate the mean shear flows (such as jets, wakes, and boundary layers) from the shear-free flows. Experiments and DNS of an "oscillating-grid" (shear-free flow) by Holzner et al. ${ }^{15,26}$ have identified the importance of viscous forces originating in strain for entrainment, and that the smallest scales are of the order of Kolmogorov length scale (e.g., da Silva and Taveira ${ }^{27}$ ). However, the results of Holzner and Lüthi ${ }^{28}$ show that the interface spreading velocity is not well correlated with the Kolmogorov velocity, even though they note that the viscous process involves a large surface area so that the global rate of turbulence spreading is set by the largest scales of motion, in agreement with the notion of a multiscale process. ${ }^{10,11}$ It is evident that the structure of turbulence and transport in the vicinity of the TNTI is associated with a range of scales. The present investigation aims to introduce and apply an analysis approach that is specifically aimed at identifying fluxes and transport at the TNTI at various scales, ranging between the largest to the smallest scales.

The paper is arranged as follows: Sec. II describes the experimental setup for the turbulent boundary layer experiments as well as the method for the detection of the TNTI from the velocity fields; Sec. III develops relevant theoretical background to describe the evolution of the total kinetic energy at the smallest scales governed by viscous fluxes-the nibbling process; Sec. IV introduces a multiscale formulation characterizing the fluxes across the interface (at largest scales: purely advective fluxes- to be associated with as explained later the engulfment process, and both nibbling and engulfment at in-between scales identified through a spatial filtering process); Sec. V undertakes calculation and discussion of fluxes and the physical process at multiple scales based on the experimental data; and Sec. VI summaries and draws final conclusions. Throughout the paper, $x, y$, and $z$ represent the streamwise, spanwise, and wallnormal directions, and $u, v$, and $w$ (or $u_{1}, u_{2}$, and $u_{3}$ ), the corresponding velocities. Whenever physical quantities are normalised by the viscous length and velocity scales $v / u_{\tau}$ and $u_{\tau}$, respectively, they are represented by plus, ${ }^{+}$symbols, e.g., normalised length $\Delta^{+}=\Delta /\left(v / u_{\tau}\right)$, velocity $u^{+}=u / u_{\tau}$, etc.

\section{EXPERIMENTAL SETUP AND INTERFACE DETECTION}

In this work, we apply planar PIV measurements in the wall-normal/streamwise direction to a flow for the purpose of investigating the interface characteristics at significantly higher Reynolds numbers than prior works. ${ }^{6,24}$ We examine physical transport processes across the TNTI using two experimental data sets in very high Reynolds number boundary layers, at $R e_{\delta}=U_{\infty} \delta / v \approx 2.37 \times 10^{5}$, and $4.6 \times 10^{5}$, corresponding to turbulent Reynolds numbers based on friction velocity $R e_{\tau}=u_{\tau} \delta / \nu$ $\approx 7870$ and 14500. The Taylor Reynolds number at the mean interface location ${ }^{25}$ estimated using isotropic relations and hot-wire data are $R e_{\lambda_{T}}=\lambda_{T} u^{\prime} / \nu \approx 230$ and 300. Some relevant parameters for the two databases are provided in Table I. Note that Table I also includes a smaller database of Adrian, Meinhart, and Tomkins ${ }^{29}$ at $\operatorname{Re}_{\tau}=2790\left(\operatorname{Re}_{\lambda_{T}}=125\right.$ at the mean interface location) available in the public domain, which shall be used only in reference to Figure 11 to understand the

TABLE I. Experimental parameters for the PIV databases, where $R e_{\delta}=\delta U_{\infty} / v, R e_{\theta}=\theta U_{\infty} / \nu$, and $\theta$ is the momentum thickness.

\begin{tabular}{|c|c|c|c|c|c|c|c|}
\hline$R e_{\tau}$ & $R e_{\delta}$ & $R e_{\theta}$ & $\begin{array}{c}U_{\infty} \\
{\left[\mathrm{ms}^{-1}\right]}\end{array}$ & $\begin{array}{c}u_{\tau} \\
{\left[\mathrm{ms}^{-1}\right]}\end{array}$ & $\begin{array}{c}\delta \\
{[\mathrm{m}]}\end{array}$ & $\Delta x^{+} \times \Delta z^{+}$ & No. of images \\
\hline $2790^{\mathrm{a}}$ & $0.7 \times 10^{5}$ & 6845 & 11.4 & 0.4 & 0.1 & $36 \times 25$ & 50 \\
\hline 7870 & $2.4 \times 10^{5}$ & 22400 & 10.1 & 0.33 & 0.36 & $52 \times 52$ & 1000 \\
\hline 14500 & $4.6 \times 10^{5}$ & 40800 & 20.0 & 0.63 & 0.35 & $49 \times 49$ & 1250 \\
\hline
\end{tabular}

${ }^{\text {a }}$ The dataset at $R e_{\tau}=2790$ is from Adrian, Meinhart, and Tomkins ${ }^{29}$ and is available in the public domain. 


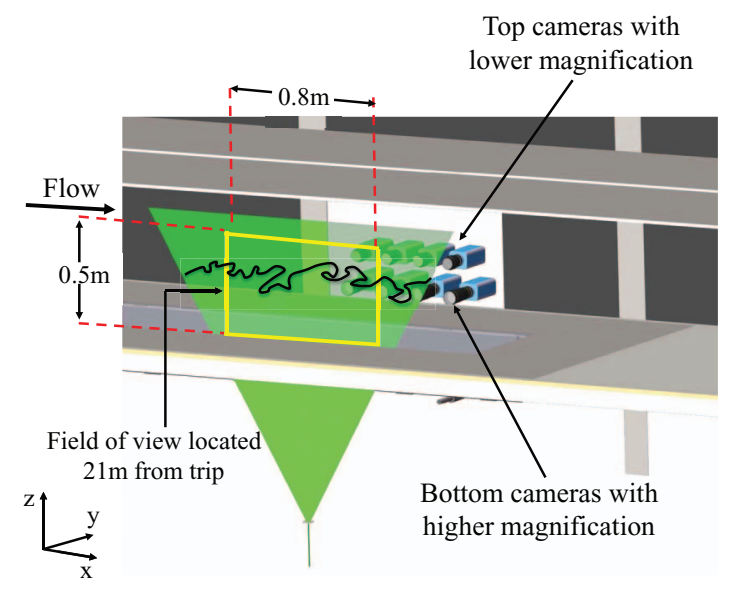

FIG. 1. Experimental setup for the planar PIV measurements using eight cameras in the High Reynolds Number Boundary Layer Wind Tunnel at the University of Melbourne.

$R e$-scaling. We note that for the different boundary layer databases, the PIV interrogation window size is about 4 to 6 times the Kolmogorov length scale in the outer region.

Figure 1 shows the experimental setup for the PIV measurements performed in the High Reynolds Number Boundary Layer Wind Tunnel (HRNBLWT) at the University of Melbourne's Walter Bassett Aerodynamics Laboratory. The region of interest is illuminated by a laser sheet in the spanwise centre of the tunnel, beamed from below the glass floor employing a Spectra Physics "QuantaRay" Nd:YAG laser rated $400 \mathrm{~mJ}$ per pulse at $532 \mathrm{~nm}$. PIV images are obtained simultaneously on eight PCO 4000 cameras $(4008 \times 2672$ pixels $)$, four cameras arranged above the rest, with the bottom cameras having a higher magnification and covering a smaller area, and an overlap of about $2 \mathrm{~cm}$ between images. The eight individual vector fields are later stitched to provide a single 2D velocity field. This provides a large dynamic range which is particularly suited to the present study where we are interested in fluxes at the smallest scales to the largest ones. Further details of the experiments (along with the comparisons of mean and turbulent statistics to hot-wire data) and processing of PIV images can be found in Chauhan et al..$^{25}$ and de Silva et al. ${ }^{30}$

Traditionally, the interface has been detected based on hot-wire velocity signals (e.g., see Ref. 31 for a comprehensive list of detector functions commonly used at that time), wherein it is not too difficult to distinguish the relatively quiet regions from those with high fluctuations. Techniques employing scalars (ideally with relatively high Schmidt number) are also popular for interface detection, both using 1D data (or a rake) from resistance thermometers (employing temperature in the turbulent region as a marker ${ }^{22}$ ) and $2 \mathrm{D}$ imaging of passive dyes in experiments ${ }^{6,10,32-34}$ as well as in Direct Numerical Simulations. ${ }^{35}$ More recently a threshold on vorticity magnitude has been used not only to distinguish turbulent from the non-turbulent regions ${ }^{12,36-38}$ primarily in DNS databases but also in moderately low Reynolds number turbulent boundary layer experiments ${ }^{24}$ where they had 3D velocity information from Tomographic-PIV measurements.

It is known (starting from the work of Corrsin and Kistler ${ }^{1}$ ) that velocity in the non-turbulent region differs from that in the turbulent region, and thus mean velocity ${ }^{1,39}$ or variance of velocity fluctuations ${ }^{40}$ have also been used for interface detection. Of the plethora of techniques available not all are suited for every type of measurement. In the present case, we have no passive scalar and velocity fields that are obtained from PIV (which are generally at a lower resolution than hot-wires and more prone to noise, especially in the non-turbulent region of the boundary layer due to large velocities there, unlike jets). Vorticity based criteria tend to include nearly irrotational islands within a fully turbulent region, which in turn leads to a highly intermittent and unconnected interface that may also be affected by the inherent "inner intermittency" within the turbulent region. Consequently, in this work we employ the kinetic energy of the flow for interface detection. In order to associate the non-turbulent outer region with zero kinetic energy, we use the kinetic energy in the frame moving 

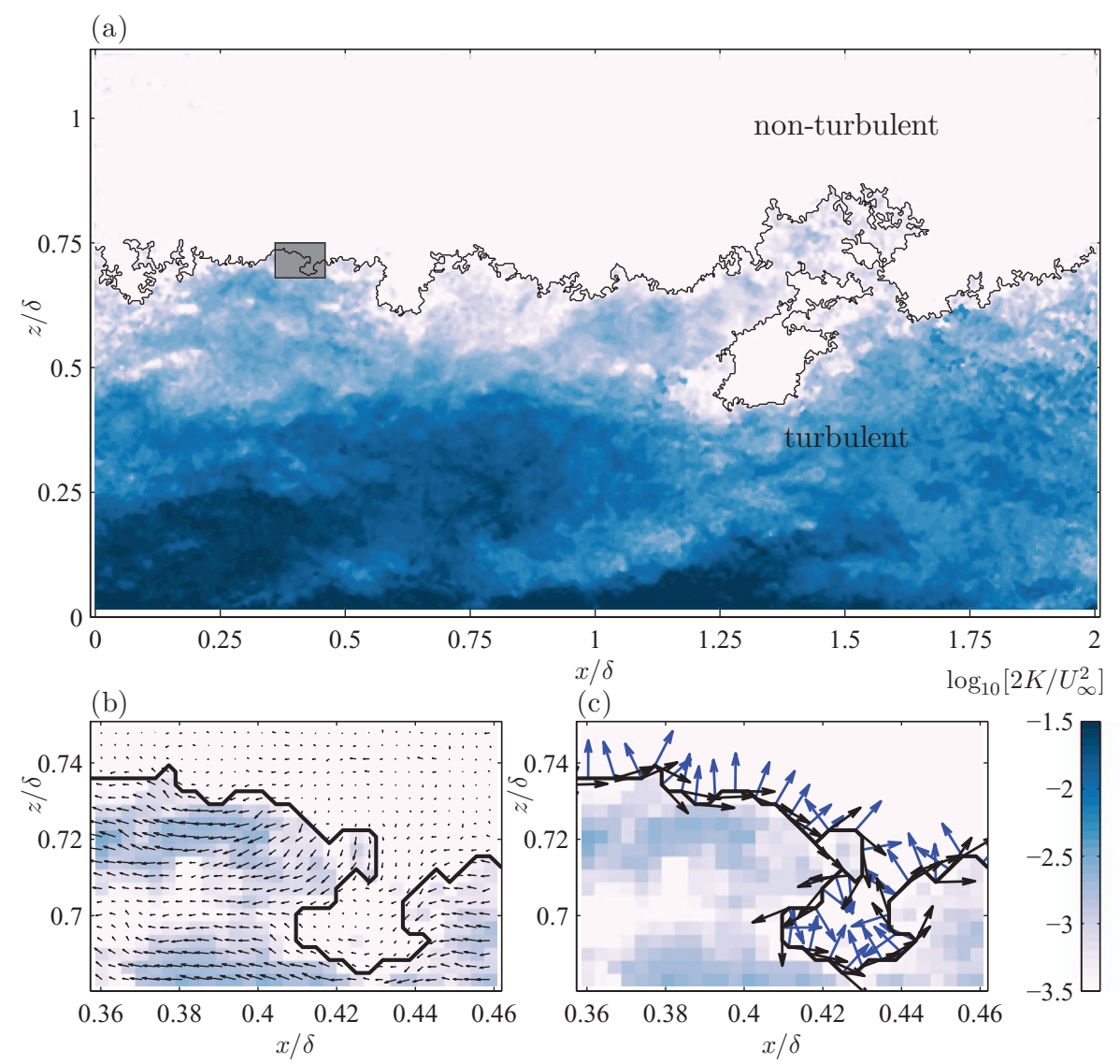

FIG. 2. Instantaneous view from planar PIV at $R e_{\tau}=14500$. (a) Colour contours of kinetic energy $K /\left(U_{\infty}^{2} / 2\right)$ in a logarithmic scale. The solid black line indicates the location of the TNTI computed as an iso-kinetic energy surface using a threshold of $K_{0}=10^{-3}\left(\frac{1}{2} U_{\infty}^{2}\right)$. (b) Zoomed in view of (a) presented as a grey region from $0.36<x / \delta<0.46$ and $0.68<y / \delta<0.75$ along with the velocity vector map in a frame moving with the free-stream. (c) The same zoomed view as in (b) with normals to the interface $\mathbf{n}$ defined by $\mathbf{n}=-(\nabla K /|\nabla K|)_{K=K_{0}}$ (in blue), and the corresponding tangent vectors $(\mathbf{j} \times \mathbf{n})$ in black.

with the free-stream, i.e., the defect kinetic energy. The same technique was employed in the fractal analysis reported in de Silva et al. ${ }^{19}$

The defect kinetic energy (its 2D surrogate) is defined according to

$$
K=\frac{1}{2}\left[\left(u-U_{\infty}\right)^{2}+w^{2}\right],
$$

where $U_{\infty}$ is the free-stream velocity in the $x$ direction. When $u$ is expressed in a frame moving with the free-stream (in a frame in which $U_{\infty}=0$ ), the expression reduces to $K=(1 / 2)\left(u^{2}+w^{2}\right)$. Figure 2(a) shows a sample PIV image and the interface determined based on the local defect kinetic energy $K$, using a threshold of $K=10^{-3}\left(\frac{1}{2} U_{\infty}^{2}\right)$. This threshold yields an interface that agrees very well with the location that visually can be observed to separate turbulent from non-turbulent flow. The appropriate threshold to be used depends on the flow, as well as on the level of freestream (background) turbulence and measurement noise. In the present case random error in the PIV measurements in the outer irrotational region is estimated to be about $1 \%$ of the free-stream velocity employing an uncertainty of 0.15 pixel movement. ${ }^{41}$ In addition, the wind-tunnel freestream turbulence intensity is about $0.2 \%$. A change in threshold value by up to $20 \%$ has been found to have negligible effect on the computed numerical values (see Sec. V) and the general conclusions are insensitive to an even bigger change. Threshold values similar to the present case have also been employed by de Silva et al. ${ }^{19}$ and Chauhan et al. ${ }^{25}$ to characterise various interface properties such as the fractal dimension, intermittency, and conditional averages. 
Figures 2(b) and 2(c) show a zoomed in view of a portion of the interface (as indicated by the grey region in Figure 2(a)) along with velocity vectors, and local normals $\left(\mathbf{n}=-\nabla K /|\nabla K|_{K=K_{0}}\right.$, where $K_{0}$ is the threshold level) and tangents to the interface defined as $\mathbf{t}=\mathbf{j} \times \mathbf{n}$, where $\mathbf{j}$ is the unit vector in the $y$-direction perpendicular to the measurement plane. Notice that, in general, (as well as in Figure 2(a)) one can also observe patches of non-turbulent regions inside the turbulent region. With the present PIV measurements, we cannot ascertain if these patches are connected to the outside non-turbulent region three-dimensionally or if they are genuinely disconnected volumes of non-turbulent regions. In any case, we shall not include these patches in the analysis when performing averages over the interface, and we will consider TNTI as the longest boundary that separates the main turbulent region from the outside non-turbulent region. Finally, we remark that (albeit for jets) based on DNS and experimental data there is evidence that using different interface detection criteria do not significantly change the interface characteristics. ${ }^{39}$

\section{KINETIC ENERGY EQUATION: FLUXES AT THE SMALLEST SCALES}

Having observed that kinetic energy relative to the free stream provides a meaningful criterion to determine the TNTI, we now turn to the dynamics and transport processes associated with kinetic energy. We consider the transport of defect kinetic energy $K(\mathbf{x}, t)$, defined according to

$$
K(\mathbf{x}, t)=\frac{1}{2}[\mathbf{u}(\mathbf{x}, t)-\mathbf{U}]^{2},
$$

where $\mathbf{U}$ is the constant velocity in the free-stream far outside the boundary layer. That is, in a traditional frame of reference attached to the wall and a free-stream moving in the positive direction ( $x$-direction with unit vector $\mathbf{i}$ ), we have $\mathbf{U}=U_{\infty} \mathbf{i}$ ( $=U_{1} \mathbf{i}$ depending on the context), while in a frame moving with the free-stream velocity in which the wall moves, we have $\mathbf{U}=0$. Note that $K(\mathbf{x}, t)$ includes the kinetic energy in both the mean flow defect velocity field, as well as all the kinetic energy in the turbulence since no averaging has yet been performed.

The momentum equation for a Newtonian incompressible flow (with viscous stress tensor $\tau_{i j}^{v}=2 v S_{i j}$, where $S_{i j}$ is the strain-rate tensor), written for the momentum defect with respect to a constant free stream velocity $\mathbf{U}$, is

$$
\frac{\partial}{\partial t}\left(u_{i}-U_{i}\right)+u_{j} \frac{\partial}{\partial x_{j}}\left(u_{i}-U_{i}\right)=-\frac{1}{\rho} \frac{\partial p}{\partial x_{i}}+\frac{\partial \tau_{i j}^{\nu}}{\partial x_{j}} .
$$

Multiplying by the velocity defect, $u_{i}-U_{i}$, results in the equation for $K(\mathbf{x}, t)$ :

$$
\frac{\partial K}{\partial t}+\frac{\partial}{\partial x_{i}}\left(u_{i} K\right)=\frac{\partial}{\partial x_{i}}\left(-\left(u_{j}-U_{j}\right) \frac{p}{\rho} \delta_{i j}+\left(u_{j}-U_{j}\right) \tau_{j i}^{\nu}\right)-\tau_{i j}^{\nu} \frac{\partial u_{j}}{\partial x_{i}} .
$$

Now consider a boundary layer control volume $\left(V_{b l}\right)$ including as its top surface the TNTI as sketched in Figure 3(a). The interface is defined as a $K=K_{0}$ iso-surface, and the dashed line is instantaneously coincident with the interface (fixed, not moving with the interface). For a boundary layer, we have used $K_{0}=c_{0} \frac{1}{2} U_{\infty}^{2}$ and we specify the (small) dimensionless factor $c_{0}$, e.g., $c_{0}=$ $10^{-3}$ as was used in de Silva et al. ${ }^{19}$ and Figure 2. The kinetic energy equation for the total kinetic energy in the boundary layer $\mathcal{K}_{V_{b l}}=\int_{V_{b l}} K d^{3} \mathbf{x}$ in the (fixed) control volume reads

$$
\frac{d \mathcal{K}_{V_{b l}}}{d t}=-\iint_{S_{b l}}\left[u_{i} K-2 v S_{i j}\left(u_{j}-U_{j}\right)\right] n_{i} d S-\iint_{S_{b l}}\left(\frac{p}{\rho}\right)\left(u_{i}-U_{i}\right) n_{i} d S-\iiint_{V_{b l}} \varepsilon d V,
$$

where $\varepsilon=\tau_{i j}^{v} \partial u_{j} / \partial x_{i}$ is the viscous dissipation rate. The advective flux can be further decomposed, and the kinetic energy equation is rewritten as follows:

$$
\begin{aligned}
\frac{d \mathcal{K}_{V_{b l}}}{d t}= & -\iint_{S_{b l}} K U_{i} n_{i} d S-\iint_{S_{b l}}\left(K+\frac{p}{\rho}\right)\left(u_{i}-U_{i}\right) n_{i} d S \\
& -\left(-2 v \iint_{S_{b l}} S_{i j}\left(u_{j}-U_{j}\right) n_{i} d S\right)-\iiint_{V_{b l}} \varepsilon d V .
\end{aligned}
$$


(a)

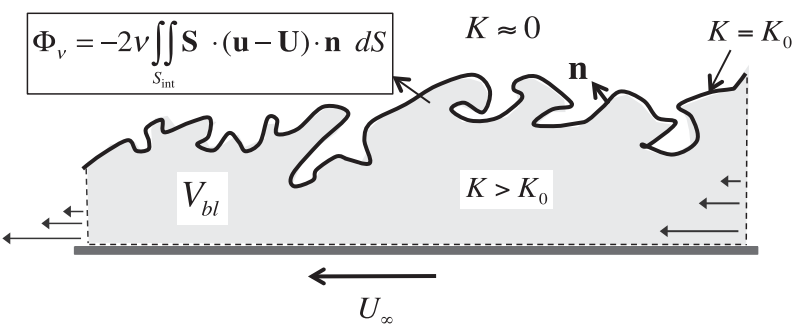

(b)

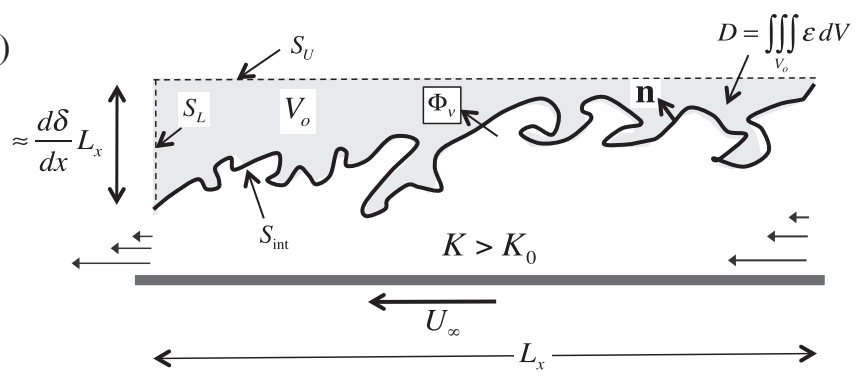

FIG. 3. Sketch of turbulent region bounded by an iso-kinetic energy surface as surrogate marker for the turbulent-non turbulent interface. (a) Kinetic energy analysis for "inner control volume," $V_{b l}$. (b) Outer control volume $V_{o}$. The mean boundary layer thickness is $\delta$.

We now concentrate on the surface integral over the interface portion of the bounding surface, $S=$ $S_{\text {int }}$, where the unit normal is given by $\mathbf{n}=-(\nabla K /|\nabla K|)_{K=K_{0}}$. The flux due to viscous work at the interface is given by

$$
\Phi_{v}=-2 v \iint_{S_{\mathrm{int}}}\left[S_{i j}\left(u_{j}-U_{j}\right)\right] n_{i} d S
$$

At this stage, it is instructive to consider the control volume formed by the "outer" domain, denoted by $V_{o}$ in Figure 3(b). It is bounded by a closed surface $S_{o}$, which consists of the interface $S_{\text {int }}$ below, a flat horizontal surface above and a vertical surface on the left. Hence, for this control volume we can write, using the same definition of $\mathbf{n}$ as before on the interface (i.e., $\mathbf{n}$ points into $V_{o}$ ),

$$
\frac{d \mathcal{K}_{V_{o}}}{d t}=\iint_{S_{o}} K U_{i} n_{i} d S+\iint_{S_{o}}\left(K+\frac{p}{\rho}\right)\left(u_{i}-U_{i}\right) n_{i} d S+\Phi_{v}-D,
$$

where $D=\iiint_{V_{o}} \varepsilon d V$ is the total dissipation rate in the outer control volume. In Appendix A, it is shown that the closed-surface integral containing the inertial terms is very small, i.e.,

$$
\iint_{S_{o}}\left(K+\frac{p}{\rho}\right)\left(u_{i}-U_{i}\right) n_{i} d S \approx 0
$$

This result assumes that the rate of change of kinetic energy inside $V_{o}$ associated with outer but weak "irrotational fluctuations" can be neglected (see Appendix A). This step shows that the flux of defect kinetic energy across $S_{\text {int }}$ due to fluctuating turbulence is largely cancelled by pressure work due to fluctuating pressure. As shown in Appendix A, if there is a difference it contributes only to irrotational kinetic energy in $V_{o}$, which is assumed to be small.

The term $\iint_{S_{o}} K U_{i} n_{i} d S$ is further simplified using $\mathbf{U}=U_{1} \mathbf{i}$ (and thus $U_{i} n_{i}=U_{1} n_{1}$ ), and neglecting $K$ on the outer surfaces except at the interface, one obtains $\iint_{S_{o}} K U_{i} n_{i} d S \approx K_{0} U_{1} \iint_{S_{\text {int }}} n_{1} d S$. (In Appendix A, we consider $U_{1} \iint_{S_{L}} K n_{1} d S$ again, but including the small amount of kinetic energy outside the interface in $V_{o}$, where $K<K_{0}$ but still $K>0$ possibly due to irrotational fluctuations.) The integral $\iint_{S_{\text {int }}} n_{1} d S$ is simply the projected area of the interface along the $x$-direction, i.e., 
$\iint_{S_{I}} n_{1} d S \approx-d \delta / d x A_{x y}$ where $A_{x y}$ is the footprint area of the control volume. The negative sign arises because $\mathbf{n}$ points into $V_{o}$ and thus $n_{1}<0$ on average if $d \delta / d x>0$ ).

Finally, we obtain

$$
\frac{d \mathcal{K}_{V_{o}}}{d t}+U_{1} K_{0} \frac{d \delta}{d x} A_{x y}=\Phi_{v}-D
$$

Note that the integrated quantity $\mathcal{K}_{V_{o}}$ represents kinetic energy in the full defect velocity field, including mean flow and turbulent fluctuations. In a frame moving with the free-stream velocity, $U_{1}$ $=0$, thus the term $U_{1} K_{0} A_{x y} d \delta / d x$ vanishes, and $d \mathcal{K}_{V_{o}} / d t$ is expected to be positive (increase of kinetic energy in the outer volume $V_{o}$ in time). On the other hand, in a frame attached to the wall, on average we have $\left\langle d \mathcal{K}_{V_{o}} / d t\right\rangle=0$ due to stationarity, but it is replaced by the term $U_{1} K_{0} d \delta / d x A_{x y}$ (recall that $U_{1}=U_{\infty}$ ), which represents the positive advective flux of $K_{0}$ due to the free-stream velocity. In a sense, this term then "counteracts" the viscous diffusion $\Phi_{v}$ "moving back defect kinetic energy" into the control volume and out of $V_{o}$ along the interface. The dissipation $D$ is expected to be small in the outer region. Thus Eq. (10) shows that the growth rate $\left\langle d \mathcal{K}_{V_{o}} / d t\right\rangle$ or $U_{1} K_{0} \frac{d \delta}{d x} A_{x y}$ of defect kinetic energy in the turbulent boundary layer is caused by the viscous flux at the interface, $\Phi_{\nu}$.

Our aim later in the paper is to examine $\Phi_{\nu}$ from PIV data. A two-dimensional surrogate that can be determined from planar PIV data (in the $x_{1}-x_{3}$ plane) along the intersection of the interface with the measurement plane is:

$$
\Phi_{v}^{2 D}=-2 v \int_{s=0}^{s_{f}}\left(S_{i j} u_{j}\right) n_{i} d s, \quad i, j=1,3,
$$

involving only two components and where the integration is performed along an interface-following coordinate $s$ that goes from the beginning of the interface at $s=0$ to the end $s=s_{f}$.

\section{MULTISCALE PROPERTIES OF FLUXES AT THE TNTI}

In order to examine multiscale properties of entrainment/nibbling/fluxes at the TNTI defined in terms of kinetic energy, we consider the kinetic energy field associated with descriptions at various scales.

\section{A. Mean flow: Ensemble (Reynolds) averaged formulation}

We begin by the description at the level of Reynolds Average Navier-Stokes (RANS) and ensemble averaging, i.e., emphasizing processes occurring at the level of the mean flow and the largest scales of turbulence. At this level, surfaces are essentially flat, growing slowly like the mean boundary layer. Denoting ensemble average by an over-bar, the kinetic energy in the mean defect flow is defined as $K^{R}=\frac{1}{2}\left(\bar{u}_{i}-U_{i}\right)^{2}$. The superscript " $\mathrm{R}$ " refers to Reynolds averaging (see Figure $4(\mathrm{c}))$. The fate of kinetic energy in the mean defect flow is governed by

$$
\begin{aligned}
\frac{\partial K^{R}}{\partial t}+\frac{\partial}{\partial x_{i}}\left(\bar{u}_{i} K^{R}\right)= & -\frac{\partial}{\partial x_{i}}\left(\frac{1}{\rho}\left(\bar{u}_{j}-U_{j}\right) \bar{p} \delta_{i j}-\left(\bar{u}_{j}-U_{j}\right) \bar{\tau}_{j i}^{v}+\left(\bar{u}_{j}-U_{j}\right) \overline{u_{i}^{\prime} u_{j}^{\prime}}\right) \\
& -\bar{\tau}_{j i}^{v} \frac{\partial \bar{u}_{j}}{\partial x_{i}}+\overline{u_{i}^{\prime} u_{j}^{\prime}} \frac{\partial \bar{u}_{j}}{\partial x_{i}} .
\end{aligned}
$$

However, there is also turbulent kinetic energy $k^{R}=\frac{1}{2} \overline{u_{i}^{\prime} u_{i}^{\prime}}$ (where $u_{i}^{\prime}=u_{i}-\bar{u}_{i}$ ), which should be added to $K^{R}$ to obtain total kinetic energy $\bar{K}=K^{R}+k^{R}$ that can be compared to the total kinetic energy considered in Sec. III.

The transport equation for turbulent kinetic energy $k^{R}=\frac{1}{2} \overline{u_{i}^{\prime} u_{i}^{\prime}}$ reads

$$
\frac{\partial k^{R}}{\partial t}+\frac{\partial}{\partial x_{i}}\left(\bar{u}_{i} k^{R}\right)=-\frac{\partial}{\partial x_{i}}\left(\frac{1}{\rho} \overline{u_{j}^{\prime} p^{\prime}} \delta_{i j}-\overline{\tau_{j i}^{\prime v} u_{j}^{\prime}}+\frac{1}{2} \overline{u_{j}^{\prime 2} u_{i}^{\prime}}\right)-\overline{u_{i}^{\prime} u_{j}^{\prime}} \frac{\partial \bar{u}_{j}}{\partial x_{i}}-\bar{\varepsilon}+\bar{\tau}_{j i}^{v} \frac{\partial \bar{u}_{j}}{\partial x_{i}} .
$$


(a) Fully resolved

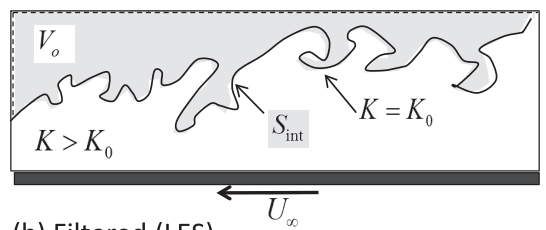

(b) Filtered (LES)

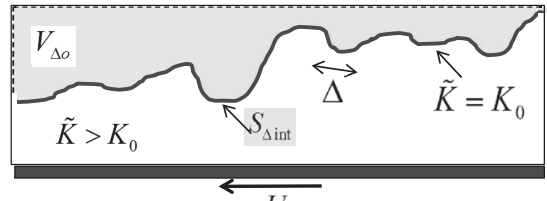

(c) Averaged (RANS)

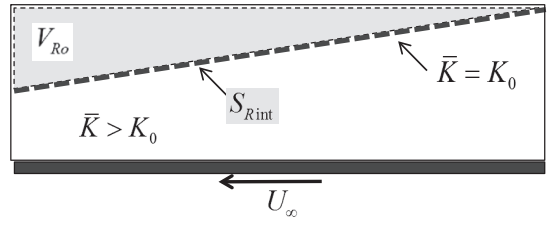

FIG. 4. Sketch of turbulent region bounded by iso-kinetic energy surface at various scales. (a) Original scale, resolving viscous stresses, (b) filtered at intermediate scale $\Delta$, (c) ensemble averaged (RANS) based on mean flow.

The transport equation for the total kinetic energy can be obtained by adding both equations, i.e.,

$$
\frac{\partial \bar{K}}{\partial t}+\bar{u}_{i} \frac{\partial \bar{K}}{\partial x_{i}}=-\frac{\partial}{\partial x_{i}}\left(\frac{1}{\rho}\left[\left(\bar{u}_{j}-U_{j}\right) \bar{p}+\overline{u_{j}^{\prime} p^{\prime}}\right] \delta_{i j}+\left(\bar{u}_{j}-U_{j}\right) \overline{u_{i}^{\prime} u_{j}^{\prime}}+\frac{1}{2} \overline{u_{j}^{\prime 2} u_{i}^{\prime}}\right)-\bar{\varepsilon},
$$

where viscous fluxes have been neglected since at high Reynolds numbers away from the wall they are expected to be small.

The interface associated with the mean flow is now defined as where the total energy $\bar{K}=K_{0}$, using the same threshold as in Secs. II and III. The unit vector is defined as $\overline{\mathbf{n}} \equiv-(\nabla \bar{K} /|\nabla \bar{K}|)_{\bar{K}}=K_{0}$, which differs from taking the average of the instantaneous $n_{i}$ due to nonlinearities in the definitions of kinetic energy and the division by the absolute value of the gradient. This definition can be naturally generalized to other scales later on and has a clear physical interpretation in terms of the kinetic energy in the flow. For example, see Appendix B (Figure 12) for a comparison of different methods to identify the interface with the same threshold value. The threshold $K_{0}$ to be employed will be quite small and thus it will define the "outer skin" of the turbulent region. Such a surface can be considered a "RANS-level interface" separating turbulence from non-turbulence, even though the interface region is expected to be quite "thick," on the order of the outer integral scale $\delta$. Thus, one has to consider the "RANS-level interface" at large scales to be able to interpret it as a "sharp" interface.

Following similar steps as in Sec. III, we define inner and outer control volumes, separated by a (smooth) interface. For the outer control volume (denoted as $V_{R o}$ ), the total energy $\overline{\mathcal{K}}_{V_{R o}}=$ $\iiint_{V_{R o}} \bar{K} d V$, obeys:

$$
\frac{d \overline{\mathcal{K}}_{V_{R o}}}{d t}+U_{1} K_{0} \frac{d \delta}{d x} A_{x y}=\iint_{S_{R o}}\left(K+\frac{\bar{p}}{\rho}\right)\left(\bar{u}_{i}-U_{i}\right) \bar{n}_{i} d S+\Phi_{A}-D_{R},
$$

where,

$$
\Phi_{A}=\iint_{S_{\text {Rint }}}\left[\left(\bar{u}_{j}-U_{j}\right) \overline{u_{i}^{\prime} u_{j}^{\prime}}+\frac{1}{2} \overline{u_{j}^{\prime 2} u_{i}^{\prime}}+\frac{1}{\rho} \overline{u_{i}^{\prime} p^{\prime}}\right] \bar{n}_{i} d S,
$$

is the flux of kinetic energy across the interface due to turbulent motions (including advection by turbulent fluctuations and pressure fluctuations), affecting the budget of kinetic energy in the mean 
defect flow, and

$$
D_{R}=\iiint_{V_{R o}} \bar{\varepsilon} d V
$$

A "Bernoulli"-like argument, in which we assume that $\bar{K}+\bar{p} / \rho \approx p_{\infty} / \rho$ (for RANS a steady Bernoulli framework is assumed to be appropriate in the irrotational region) will lead to $\iint_{S_{R o}}\left(K+\frac{\bar{p}}{\rho}\right)\left(\bar{u}_{i}-U_{i}\right) \bar{n}_{i} d S \approx 0$. Hence,

$$
\frac{d \overline{\mathcal{K}}_{V_{R o}}}{d t}+U_{1} K_{0} \frac{d \delta}{d x} A_{x y}=\Phi_{A}-D_{R}
$$

The flux $\Phi_{A}$ can be considered to be a definition of the "entrainment-rate" by the large scales since the Reynolds stress tensor and turbulent transport is dominated by the large scales. We shall also identify this term as corresponding to "large-scale engulfment" since it is associated with the Reynolds stresses that depend mostly on the energy-containing eddies.

And, since the left-hand side of (18) is the same as that in (10) for the total energy in the instantaneous description, and the dissipation terms are also the same, it follows that the fine-grained and coarse-grained fluxes should be equal, i.e., $\Phi_{A}=\Phi_{\nu}$, at least in the averaged sense.

At low Reynolds numbers, the viscous fluxes from the mean flow should be added to $\Phi_{A}$. Considering Figure 4(a), the corresponding interface is shown with the thick dashed line, assuming it to be almost linear, i.e., with an angle $\theta_{b l} \approx \tan ^{-1}(d \delta / d x)$.

\section{B. Filtered flow: LES formulation}

Next, we consider turbulence at various scales, using "coarse-grained" filtered velocities as is usually applied in Large Eddy Simulations (LES). Consider the kinetic energy in turbulence at scales equal to and larger than some scale $\Delta: K^{\Delta}=\frac{1}{2} \tilde{u}_{i} \tilde{u}_{i}$. The "tilde" refers to, for example, a box filtering, i.e., a convolution of the velocity components with a top-hat filter function $G_{\Delta}$, namely $\tilde{u}_{i} \equiv G_{\Delta} * u_{i}$. This defines a kinetic energy field that is smoother than the original. The evolution equation for $K^{\Delta}(\mathbf{x}, t)$ is obtained by starting from the filtered momentum equation, ${ }^{42,43}$ written here as before for the (filtered) velocity defect:

$$
\frac{\partial\left(\tilde{u}_{i}-U_{i}\right)}{\partial t}+\tilde{u}_{k} \frac{\partial\left(\tilde{u}_{i}-U_{i}\right)}{\partial x_{k}}=-\frac{1}{\rho} \frac{\partial \tilde{p}}{\partial x_{i}}+\frac{\partial \tilde{\tau}_{i j}^{v}}{\partial x_{j}}-\frac{\partial \tau_{i j}^{\Delta}}{\partial x_{j}},
$$

where $\tau_{i j}^{\Delta}=\widetilde{u_{i} u_{j}}-\tilde{u}_{i} \tilde{u}_{j}$ is the "subgrid-scale" (SGS) or "subfilter-scale" stress tensor. Instantaneous fields of SGS stress tensor in 2D planes can be obtained from PIV data simply by box-filtering the product $u_{i} u_{j}$ (e.g., $i, j=1,3$, in vertical wall-normal planes in boundary layers) and at each point subtracting the product of the filtered velocities. ${ }^{44-46}$ Thus in the present case, one obtains three spatial fields for $\tau_{11}^{\Delta}\left(x, z, t_{0}\right), \tau_{33}^{\Delta}\left(x, z, t_{0}\right)$, and $\tau_{13}^{\Delta}\left(x, z, t_{0}\right)$.

Multiplying (19) by $\left(\tilde{u}_{i}-U_{i}\right)$ leads to the equation for $K^{\Delta}(\mathbf{x}, t)=\frac{1}{2}\left(\tilde{u}_{i}-U_{i}\right)^{2}$, the kinetic energy in the large-scale field of the velocity defect:

$$
\frac{\partial K^{\Delta}}{\partial t}+\frac{\partial}{\partial x_{i}}\left(\tilde{u}_{i} K^{\Delta}\right)=-\frac{\partial}{\partial x_{i}}\left(\frac{1}{\rho}\left(\tilde{u}_{j}-U_{j}\right) \tilde{p} \delta_{i j}-\left(\tilde{u}_{j}-U_{j}\right) \tilde{\tau}_{i j}^{v}+\left(\tilde{u}_{j}-U_{j}\right) \tau_{i j}^{\Delta}\right)-\tilde{\tau}_{i j}^{v} \tilde{S}_{i j}+\tau_{i j}^{\Delta} \tilde{S}_{i j} .
$$

There is also kinetic energy contained in the SGS motions, $k^{\Delta}=\frac{1}{2}\left(\widetilde{u_{i} u_{i}}-\tilde{u}_{i} \tilde{u}_{i}\right)$, and its transport equation is given by

$$
\begin{aligned}
{\left[\frac{\partial}{\partial t}+\tilde{u}_{j} \frac{\partial}{\partial x_{j}}\right] k^{\Delta}=} & -\frac{\partial}{\partial x_{j}}\left(\frac{1}{\rho}\left(\widetilde{p u_{i}}-\tilde{p} \tilde{u}_{i}\right) \delta_{i j}+\left(-\widetilde{u_{i} \tau_{i j}^{v}}+\tilde{u}_{i} \tilde{i}_{i j}^{v}\right)-\tilde{u}_{i} \tau_{i j}^{\Delta}+\right. \\
& \left.\frac{1}{2}\left(\widetilde{u_{j} u_{i} u_{i}}-\tilde{u_{j}} \widetilde{u_{i} u_{i}}\right)\right)-\widetilde{\tau_{i j}^{v} S_{i j}}+\tilde{\tau}_{i j}^{v} \tilde{S}_{i j}-\tau_{i j}^{\Delta} \tilde{S}_{i j} .
\end{aligned}
$$


The total kinetic energy in the defect field, when viewed at scale $\Delta$ is given by $\tilde{K}=K^{\Delta}+k^{\Delta}$ and its transport equation at that scale is obtained by adding the equations for $K^{\Delta}$ and $k^{\Delta}$, resulting in

$$
\begin{aligned}
{\left[\frac{\partial}{\partial t}+\tilde{u}_{j} \frac{\partial}{\partial x_{j}}\right]\left(K^{\Delta}+k^{\Delta}\right)=} & -\frac{\partial}{\partial x_{j}}\left(\frac{1}{\rho}\left(\widetilde{p u_{i}}-U_{i} \tilde{p}\right) \delta_{i j}-\left(\widetilde{u_{i} \tau_{i j}^{v}}-U_{i} \tilde{\tau}_{i j}^{v}\right)\right. \\
& \left.+\left(\widetilde{u_{j} u_{i} u_{i}}-\tilde{u}_{j} \widetilde{u_{i} u_{i}}\right) / 2-U_{i} \tau_{i j}^{\Delta}\right)-\widetilde{\tau_{i j}^{\nu} S_{i j}}
\end{aligned}
$$

We define the interface as the iso-level of $\tilde{K}=K^{\Delta}+k^{\Delta}$ at $K_{0}=c_{0} \frac{1}{2} U_{\infty}^{2}$ as before, and the unit normal is denoted according to $\tilde{\mathbf{n}}=-(\nabla \tilde{K} /|\nabla \tilde{K}|)_{\tilde{K}=K_{0}}$. Notice that, (22) reduces to (4) in the limit $\Delta \rightarrow 0$, corresponding to the unfiltered field. In the limit of large $\Delta$ with $\Delta$ exceeding the turbulence integral scale and to the degree that spatial and ensemble averaging yield similar results (this does not hold in flows with strong spatial mean inhomogeneities), the formulation tends to that of RANS, where (22) reduces to (14) (assuming as in (14) that the viscous stress gradient terms are small). Also, we recall that the notion of a "sharp interface" can be applied to the LES-level interface only when viewed at scales larger than $\Delta$, whereas, at scales smaller than $\Delta$ the interface is smeared out by construction.

We now apply the control volume argument to the total kinetic energy in the outer control volume bounded below by the filtered interface at scale $\Delta$ (see Figure 4(b) for the filtered control volume and Figures 4(a) and 4(c) for comparison with no filtering and RANS case). Defining, $\tilde{\mathcal{K}}_{V_{\Delta o}}=\iiint_{V_{\Delta o}} \tilde{K} d V$ and $\chi_{j}^{\Delta} \equiv \widetilde{p u_{j}}-\tilde{p} \tilde{u}_{j}$, we obtain

$$
\frac{d \tilde{\mathcal{K}}_{V_{\Delta o}}}{d t}+U_{1} K_{0} \frac{d \delta}{d x} A_{x y}=\iint_{S_{\Delta o}}\left(K+\frac{\tilde{p}}{\rho}\right)\left(\tilde{u}_{j}-U_{j}\right) \tilde{n}_{j} d S+\Phi_{A \Delta}+\Phi_{\nu \Delta}-D_{\Delta},
$$

where

$$
\Phi_{A \Delta}=\iint_{S_{\Delta \text { int }}}\left[\frac{1}{2}\left(\widetilde{u_{j} u_{i} u_{i}}-\tilde{u_{j}} \widetilde{u_{i} u_{i}}\right)-U_{i} \tau_{i j}^{\Delta}+\frac{1}{\rho} \chi_{j}^{\Delta}\right] \tilde{n}_{j} d S
$$

is the flux of kinetic energy across the interface due to turbulent motions at the scale $\Delta$, whereas,

$$
\left.\Phi_{\nu \Delta}=-2 v \iint_{S_{\Delta \text { int }}}\left[\widetilde{\left(u_{i} S_{i j}\right.}-U_{i} \tilde{S}_{i j}\right)\right] \tilde{n}_{j} d S
$$

is the contribution of viscous fluxes to the kinetic energy transport across the interface, and,

$$
D_{\Delta}=\iiint_{V_{\Delta o}} \tilde{\varepsilon} d V
$$

Resorting again to the Bernoulli equation as before, wherein, $(K+\tilde{p} / \rho) \approx p_{\infty} / \rho$ is assumed, leads to,

$$
\frac{d \tilde{\mathcal{K}}_{V_{\Delta o}}}{d t}+U_{1} K_{0} \frac{d \delta}{d x} A_{x y}=\Phi_{A \Delta}+\Phi_{v \Delta}-D_{\Delta}
$$

Note that in the definition of $D_{\Delta}$, the integration and the filtering commute. Hence, if the control volume $V_{\Delta o}$ is sufficiently large, it is quite reasonable to assume that all of these total rates of dissipation are essentially the same: $D \approx D_{A} \approx D_{\Delta}$, if not exactly for instantaneous fields, at least after ensemble averaging. Since the left-hand side of Eqs. (10), (18) and (27) should also be the same (neglecting fluctuations in the definitions of $\delta$ ), it follows that the averaged

$$
\Phi_{v} \approx \Phi_{A} \approx \Phi_{A \Delta}+\Phi_{\nu \Delta},
$$

regardless of $\Delta$. If only planar experimental data are available, 2D surrogates $\Phi_{A \Delta}^{2 D}$ and $\Phi_{\nu \Delta}^{2 D}$ can be defined by restricting $i, j=1,3$. Such $2 \mathrm{D}$ surrogate quantities, however, will involve missing information from "surface folds" and components in the directions normal to the data plane especially at smaller scales. At larger scales, when the flow approaches the mean which is two-dimensional, the 2D surrogate definitions are expected to be correct, but at small scales, where the flow structure is 
highly 3D, important contributions would be missing. The concept of local isotropy may be invoked at scales below some threshold, i.e., for $\Delta<\Delta_{i s o}$. However, the experimental data show that very small scales would be needed to approach such local isotropy (cf., Sec. V A and Appendix C). Such inherent limitations of 2D surrogates will need to be kept in mind when interpreting measurement results.

The above analysis is carried out for a control volume $V_{o}\left(\mathbf{x}, t=t_{0}\right)$ that is fixed at an instant in time, $t_{0}$. A similar analysis can be performed based on a deforming control volume which changes with time, $V_{d o}(t)$. The control volume $V_{d o}$ is attached to the evolving interface which moves at velocity $\mathbf{u}_{\text {int }}$. See Appendix D for the details. The approach leads to the definition of the local entrainment velocity $\mathbf{v}=\mathbf{u}-\mathbf{u}_{\text {int }}$ (e.g., Ref. 47). At any scale $\Delta$, it is shown (see Appendix D) that the total flux, $\left(\Phi_{A \Delta}+\Phi_{v \Delta}\right)$ can be used to define a surface averaged net entrainment velocity at that scale, $v_{s}^{\Delta}$, such that, $v_{s}^{\Delta} S(\Delta)=\left(\Phi_{A \Delta}+\Phi_{v \Delta}\right) / K_{0}$, where $S(\Delta)$ is the surface area at a particular scale $\Delta$. Since the total flux at any scale has to be a constant, it implies that $v_{s}^{\Delta} S(\Delta)$ is also a constant.

\section{EXPERIMENTAL RESULTS AND DISCUSSION}

Velocity fields from the PIV databases are employed to calculate the various energy fluxes across the interface. Accordingly, the surface integrals over the interface are replaced by line integrals along the wrinkled line over the contour corresponding to the interface on the 2D vector field. Fluxes are calculated in a reference frame moving with the free stream velocity, i.e., $\mathbf{U}=\mathbf{0}$. Energy fluxes for the three cases will be presented sequentially, beginning with the unfiltered case, the RANS case, and finally the filtered/LES case. Gradients are calculated using the method of least squares. ${ }^{41}$ Specifically, we employ a 2D Savitzky-Golay least square scheme with a stencil of $5 \times 5$ and a third order polynomial fitting.

Before presenting results, we note that the data analysis to obtain viscous flux $\Phi_{v}^{2 D}$ for the unfiltered case is expected to lead to under-predictions of the flux since the spacing between the data points (of $\approx 10 \eta$ to $4 \eta$, from the mean interface location to the outer edge of the boundary layer) does not extend to scales at or below the Kolmogorov length scale $(\eta)$. Therefore, the measured $\Phi_{v}^{2 D}$ is denoted by including a hat, $\hat{\Phi}_{v}^{2 D}$, to represent the resolution effect inherent in the data. For the same reason, experimentally measured $\Phi_{v \Delta}^{2 D}$ is denoted by $\hat{\Phi}_{v \Delta}^{2 D}$. Furthermore, in the case of the subgrid flux $\Phi_{A \Delta}^{2 D}$ as function of filter scale $\Delta$, the lack of data on the pressure field prevents us from measuring the subgrid pressure contribution $\chi_{j}^{\Delta}$ (although some estimates shall be provided). As a result of these limitations, the focus of the analysis will be on the general trends of measured terms, rather than their precise numerical values.

\section{A. Kinetic energy fluxes at small scales: Viscous effects}

For the unfiltered field, Eq. (10) shows that the rate of change of kinetic energy is due to viscous flux ( $\left.\Phi_{v}\right)$ across the interface and the dissipation in $V_{o}$. Figure 5 shows $\hat{\Phi}_{v}^{2 D}$, the 2D surrogate of $\Phi_{v}$ given by (11) at the resolution afforded by the experimental data for different images (in symbols). These results are for the $R e_{\tau}=14500$ data. Also shown (solid line) is the running average, showing the robust convergence of $\hat{\Phi}_{v}^{2 D}$ to its mean value with an increasing number of data sets. The value is positive, which implies that at the smallest scales the viscous fluxes are driving the entrainment. This conversion of non-turbulent fluid into turbulent fluid by viscosity can thus be interpreted as the "nibbling" process. As captured with the present measurement resolution, the mean value is $\hat{\Phi}_{v}^{2 D} \approx 6.6 \times 10^{-3} \mathrm{~m}^{4} \mathrm{~s}^{-3}$. This numerical value will have be compared with the fluxes arising at other scales in Secs. V B-V D.

The positivity of $\hat{\Phi}_{v}^{2 D}$ is robust because it not only holds for the mean but also for the individual images. To understand this further, we select a single image/field, say the first one corresponding to Figure 5 (which is the same shown in Figure 2). The viscous flux is written as:

$$
\hat{\Phi}_{v}^{2 D}=\int_{s=0}^{s_{f}} B_{i} n_{i} d s
$$




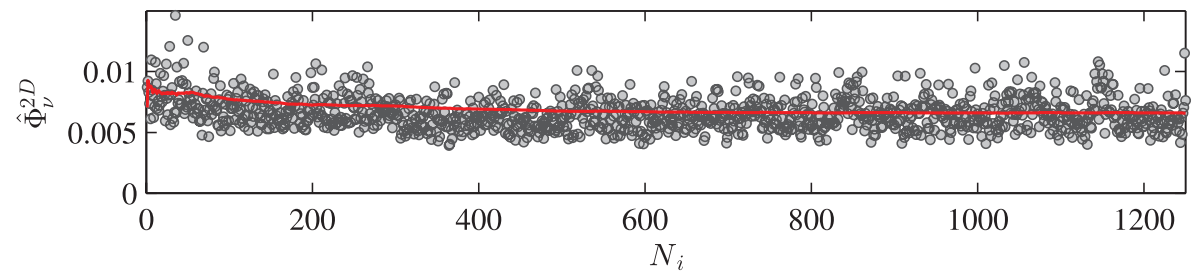

FIG. 5. Symbols: measured viscous flux of kinetic energy at the TNTI, $\hat{\Phi}_{v}^{2 D}$ (as resolved with the present spatial accuracy), for each of the 1250 vector fields at $R e_{\tau}=14500$. The solid (red) line shows the running average of $\hat{\Phi}_{v}^{2 D}$ with increasing number of images $N_{i}$. The units of the viscous flux shown are $\mathrm{m}^{4} \mathrm{~s}^{-3}$.

where, $i, j=1,3$ and $B_{i} \equiv-2 v S_{i j} u_{j}$ is the viscous flux vector across the interface at the measurement resolution. Figure 6(a) shows the instantaneous normals, $\mathbf{n}$ (blue arrows) as well as $\mathbf{B}$ (black arrows) along the interface (depicted for only a part of the image for clarity). The vector $\mathbf{B}$ is almost always pointing outwards, showing that $B_{i} n_{i}$ is almost always positive. It is consistent with the view that the viscous stresses are "nibbling" the boundary, in turn entraining the irrotational fluid. As an interesting aside we note that, if instead of the correct dissipation $\varepsilon=2 v s_{i j} s_{i j}$, we use the "pseudo-dissipation" $\epsilon^{*}=v\left(\partial u_{i} / \partial x_{i}\right)^{2}$ in (10), we would obtain a modified viscous flux, $\Phi_{v}^{*}=-v \int\left(\nabla_{i} K\right) n_{i} d S$. This in turn shows that the vector $\mathbf{B}^{*}$ is now equal to $-\nabla K$ and $\mathbf{B}^{*}$ will be exactly parallel to the interface normal, unlike in Figure 6(a) where $\mathbf{B}$ is not exactly parallel to $\mathbf{n}$. For our analysis we retain the physically more correct definition based on the true work and dissipation done by viscous forces.

It is of interest to understand the dominant terms that contribute to the "nibbling" process. To this end, notice that in the 2D $(x, z)$ co-ordinate system, $B_{i} n_{i}$ has four components when expanded $\left(S_{11} u_{1} n_{1}, S_{13} u_{3} n_{1}, S_{31} u_{1} n_{3}\right.$, and $\left.S_{33} u_{3} n_{3}\right)$, and it has been observed (not shown here) that all these terms are approximately of similar magnitude. However, it seems more reasonable to transform these components along the tangential $\left(t\right.$ or $\left.x_{t}\right)$ and the normal $\left(n\right.$ or $\left.x_{n}\right)$ directions, which leads to:

$$
B_{i} n_{i}=B_{n}=-2 v\left(S_{t n} u_{t}+S_{n n} u_{n}\right)=-2 v\left(\frac{1}{2} \frac{\partial u_{n}}{\partial x_{t}} u_{t}+\frac{1}{2} \frac{\partial u_{t}}{\partial x_{n}} u_{t}+\frac{\partial u_{n}}{\partial x_{n}} u_{n}\right),
$$

with no summation over $t, n$. The subscripts $t, n$ denote the tangential and normal components of the tensor and vectors. Evaluations from the data show that the first term on the rightmost side of (30) is about two orders of magnitude smaller than the other two which are of the same order of magnitude. This is not surprising because variations along the tangential direction are known to be negligible, ${ }^{21}$ unlike those along the normal direction where the mean velocity has a sharp variation (also called the

(a)

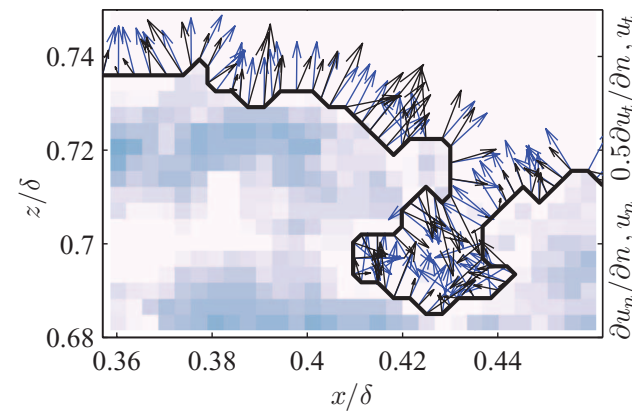

(b)

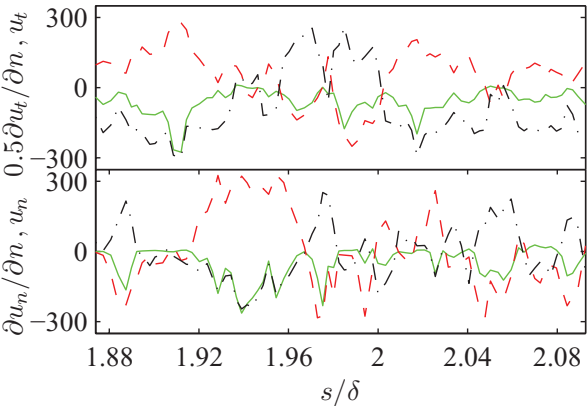

FIG. 6. Instantaneous viscous flux $\hat{\Phi}_{v}^{2 D}$ along the interface. (a) Portion of the interface (black solid line) along with normals (n) blue arrows, and the viscous flux vector $\left(\mathbf{B}\right.$, with $\left.B_{i} \equiv-2 v S_{i j} u_{j}\right)$, black arrows, with contours of kinetic energy the same as shown in Figure 2. (b) Top panel: $\left(0.5 \partial u_{t} / \partial x_{n}\right)\left[\mathrm{s}^{-1}\right]$, dashed (red) line; $\left(300 u_{t}\right)\left[\mathrm{ms}^{-1}\right.$ ], dashed-dotted (black) line; and $\left(0.5 \partial u_{t} / \partial x_{n} u_{t}\right)\left[\mathrm{ms}^{-2}\right]$ solid (green) line. Bottom panel: $\left(\partial u_{n} / \partial x_{n}\right)\left[\mathrm{s}^{-1}\right]$, dashed (red) line; $\left(300 u_{n}\right)\left[\mathrm{ms}^{-1}\right]$, dashed-dotted (black) line; and $\left(\partial u_{n} / \partial x_{n} u_{n}\right)\left[\mathrm{ms}^{-2}\right]$, solid (green) line. Note that the velocities are multiplied by a factor of 300 in (b) for clarity. 
velocity jump). Figure 6(b) shows on the top panel $\left(0.5 \partial u_{t} / \partial x_{n}\right),\left(u_{t}\right)$ and their product along the interface shown in Figure 6(a), and the bottom panel shows similar contributions of $\left(\partial u_{n} / \partial x_{n}\right)$ and $\left(u_{n}\right)$. They are calculated by first evaluating $S_{i j}$ and $u_{i}$ in the $(x, z)$ co-ordinate system and then transforming them to the $(t, n)$ system; for example, $S_{t n}=l_{i t} l_{j n} S_{i j}$ and $u_{t}=l_{i t} u_{i}$, where, $l_{i j}$ is the usual cosine matrix. It is observed from Figure $6(\mathrm{~b})$ that $\left(0.5 \partial u_{t} / \partial x_{n}\right)$ and $\left(u_{t}\right)$, as well as $\left(\partial u_{n} / \partial x_{n}\right)$ and $\left(u_{n}\right)$ are negatively correlated (for this particular image the correlation coefficient is $\approx-0.7$ for both top and bottom panels, and similar values are observed for other realisations, too), which in turn make $B_{n}$ negative. The negative correlation between the velocity and its gradient across the interface is a manifestation of the fact that, whether $u_{t}$ (or $u_{n}$ ) is positive or negative, the magnitude of $u_{t}$ (or $u_{n}$ ) is mostly smaller in the non-turbulent region compared to the turbulent region. And this higher magnitude of kinetic energy in the turbulent region is the cause for driving or diffusing the interface outwards (i.e., along $n$ ).

\section{B. Kinetic energy fluxes at large scales (RANS): Advective effects}

The RANS kinetic energy evolution given by (18) is the other extreme of the unfiltered case, wherein the fluxes are almost independent of viscosity and governed by large-scale fluctuations obtained from the ensemble average. The growth of $V_{o}$ is due to the advective flux (16), which for our 2D data (and ignoring the pressure velocity terms) is given by

$$
\Phi_{A}^{2 D}=\int_{s=0}^{s_{f}}\left(P_{i}+Q_{i}\right) \bar{n}_{i} d s
$$

where, $i, j=1,3$, and

$$
P_{i} \equiv \bar{u}_{j} \overline{u_{i}^{\prime} u_{j}^{\prime}} \quad \text { and }, \quad Q_{i} \equiv \overline{u_{j}^{\prime} u_{j}^{\prime} u_{i}^{\prime}}
$$

are the flux vectors corresponding to the advection of kinetic energy by the mean and fluctuations, respectively.

Figure 7(a) shows the ensemble averaged kinetic energy field along with the interface detected on the mean field with the same threshold of $K_{0}=0.1 \%$ as before. Note that even though the overall field looks smooth (1250 fields are employed for averaging), there are still very small undulations that are noticeable on the interface, due to lack of complete statistical convergence. Figure 7(b) is a zoomed in view of the interface which also shows vectors $\mathbf{P}$ (black) and $\mathbf{Q}$ (red), and the normal vector $\overline{\mathbf{n}}$ is upwards. Small "bumps" are still visible at very small scales in the discretely marked interface although $\overline{\mathbf{n}}$ is predominantly in the wall-normal direction (except for the small growth of the boundary layer). Calculations show that the terms $P_{3} \bar{n}_{3}$ and $Q_{3} \bar{n}_{3}$ contribute about equally to $\Phi_{A}^{2 D}$, and the other terms are about an order of magnitude smaller than these. $Q_{3}=\overline{u_{j}^{\prime} u_{j}^{\prime} u_{3}^{\prime}}$ implies that the wall-normal fluctuations carry the turbulent kinetic energy more efficiently than the streamwise fluctuation, not surprisingly because $\bar{n}_{3}$ is much greater than $\bar{n}_{1}$. Out of the two terms contributing

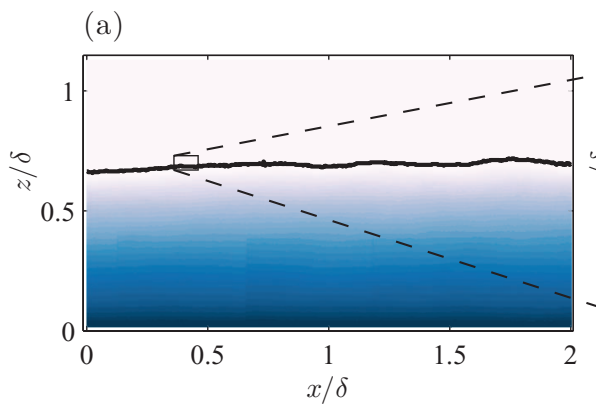

(b)

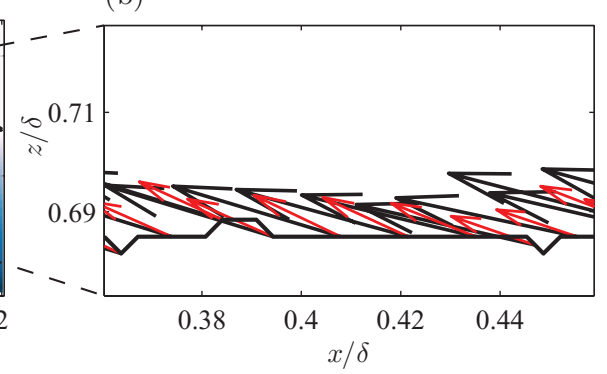

FIG. 7. Components of RANS advective flux $\Phi_{A}^{2 D}$ along the interface. (a) Ensemble averaged kinetic energy (RANS) field along with the interface at a threshold value of $K_{0}=0.1 \%$, with contours of kinetic energy the same as shown in Figure 2 . (b) Portion of the interface zoomed in from (a) along with vectors (skipping every four vectors for clarity); the mean advection flux vector $\left(\mathbf{P}\right.$, with $\left.P_{i} \equiv \bar{u}_{j} \overline{u_{i}^{\prime} u_{j}^{\prime}}\right)$, black arrows; and the turbulent advection flux vector $\left(\mathbf{Q}\right.$, with $\left.Q_{i} \equiv \overline{u_{j}^{\prime} u_{j}^{\prime} u_{i}^{\prime}}\right)$, red thin arrows. 
to $P_{3} \bar{n}_{3}, \bar{u}_{1} \overline{u_{1}^{\prime} u_{3}^{\prime}} \bar{n}_{3}$ dominates, implying that mean streamwise velocity in conjunction with the Reynolds stress at the interface also contribute significantly to the entrainment. It is interesting to note that the inviscid analysis of Reynolds ${ }^{21}$ shows that there is a "jump" across the interface in the Reynolds stress which is equal to the product of the mean normal velocity across the interface and the "jump" in the mean tangential velocity. This shows that $P_{3}$ can in turn be related to the mean effect. However, we show that fluctuations also have an equally important role to play in the large-scale entrainment via the term $Q_{3}$.

\section{Kinetic energy fluxes at intermediate (filtered) scales: Viscous/advective effects}

For a multi-scale analysis of the entrainment, the PIV database is coarse-grained using spatial filtering and contributions from viscous and advective fluxes are quantified. The velocity field and nonlinear terms are filtered with a kernel $G_{\Delta}(\mathbf{x})$, such that, e.g., $\tilde{u}_{i}=\int u_{i}\left(\mathbf{x}-\mathbf{x}^{\prime}\right) G_{\Delta}\left(\mathbf{x}^{\prime}\right) d^{2} \mathbf{x}^{\prime}$. In the present case, we employ a $2 \mathrm{D}$ box-filter $G_{\Delta}(x, z)=1 / \Delta^{2}$ if $|x|,|z|<\Delta / 2$ and $G_{\Delta}(x, z)=0$ otherwise.

For the PIV data, 2D surrogates of LES advective (24) and viscous (25) fluxes are defined respectively as

$$
\Phi_{A \Delta}^{2 D}=\int_{s=0}^{s_{f}} \tilde{A}_{i} \tilde{n}_{i} d s \quad \text { and, } \quad \hat{\Phi}_{\nu \Delta}^{2 D}=\int_{s=0}^{s_{f}} \tilde{B}_{i} \tilde{n}_{i} d s
$$

where, the LES advective flux vector $\tilde{A}_{j} \equiv(1 / 2)\left(\widetilde{u_{j} u_{i} u_{i}}-\tilde{u}_{j} \widetilde{u_{i} u_{i}}\right)$, and the corresponding viscous flux vector $\tilde{B}_{j} \equiv-2 v \widetilde{u_{i} S_{i j}}$. Figures 8 (a) and 8 (b) show two examples of filtering at filter sizes of $\Delta^{+}=150$ and 2250 , respectively. We recall that the spacing between the vectors is about 50 viscous units. The effect of filtering is clearly visible in the top panel, with small scale features smoothed out for higher filter size. The viscous flux vector has essentially the same characteristics for both filter sizes (as noticed in connection with the unfiltered case); $\tilde{\mathbf{B}}$ is mostly facing outwards in the direction of $\tilde{\mathbf{n}}$, even though the magnitudes have dropped drastically with filtering. On the other hand, the advective flux vectors $\tilde{\mathbf{A}}$ have much higher magnitudes than $\tilde{\mathbf{B}}$, and are generally directed in the negative $x$-direction. For smaller filter sizes (cf., Figure 8(a), middle panel) $\tilde{\mathbf{A}}$ has no preferential alignment with $\tilde{\mathbf{n}}$; for larger filter sizes (cf., Figure 8(b), middle panel) $\tilde{\mathbf{A}}$ is more uniform and still not aligned with $\tilde{\mathbf{n}}$; however, now $\tilde{\mathbf{A}}$ might seem better correlated with $\tilde{\mathbf{n}}$ simply because the interface is smoother. In fact, $\left(1 / l_{s}\right) \int(\widehat{\tilde{\mathbf{A}}} \cdot \tilde{\mathbf{n}}) d s$ (where, ${ }^{\wedge}$ denotes the unit vector) increases from 0.01 to 0.03 for $\Delta^{+}$increasing from 150 to 2250 for the data in Figure 8, whereas, $\left(1 / l_{s}\right) \int(\widehat{\tilde{\mathbf{B}}} \cdot \tilde{\mathbf{n}}) d s \approx 0.9$ for both filter sizes.

Consequently, advective and "resolved" viscous fluxes contribute differently to the entrainment (or in this case, to the outward growth of the interface) at various scales. Advective fluxes are dictated by the large-scale features of the flow, and not by the local interface, and rely on their large magnitudes (rather than alignment with local normal) to drive the interface outwards. On the other hand, viscous flux vectors are largely aligned with the local interface normally and drive the interface, notwithstanding the fact that their magnitudes decrease with increasing filter size, thus becoming less effective at higher $\Delta^{+}$.

Calculations of $\Phi_{A \Delta}^{2 D}$ and $\hat{\Phi}_{v \Delta}^{2 D}$ for individual fields for the $R e_{\tau}=14500$ data are shown in Figures 9(a) and 9(b), respectively, for $\Delta^{+}=150$ and 2250. The convergence with $N_{i}$ to their respective mean values occurs relatively quickly for both $\Phi_{A \Delta}^{2 D}$ and $\hat{\Phi}_{v \Delta}^{2 D}$. Note that the first data points in Figure 9 correspond to the fields shown in Figure 8.

\section{Comparison of unfiltered and RANS with multi-scale (LES) fluxes}

Figure 10 shows the viscous (unfiltered) flux $\hat{\Phi}_{v}^{2 D}$, the advective RANS flux $\Phi_{A}^{2 D}$, and the individual LES fluxes $\hat{\Phi}_{v \Delta}^{2 D}$ and $\Phi_{A \Delta}^{2 D}$, as well as their sum as function of filter scale, for the $R e_{\tau}=14500$ data in dark symbols. (The lighter symbols are for different threshold levels to be discussed below.) The dashed line represents the value of $\Phi_{A}^{2 D}$. The data points for the unfiltered and multi-scale fluxes are first calculated for individual images and subsequently averaged over all 
(a)
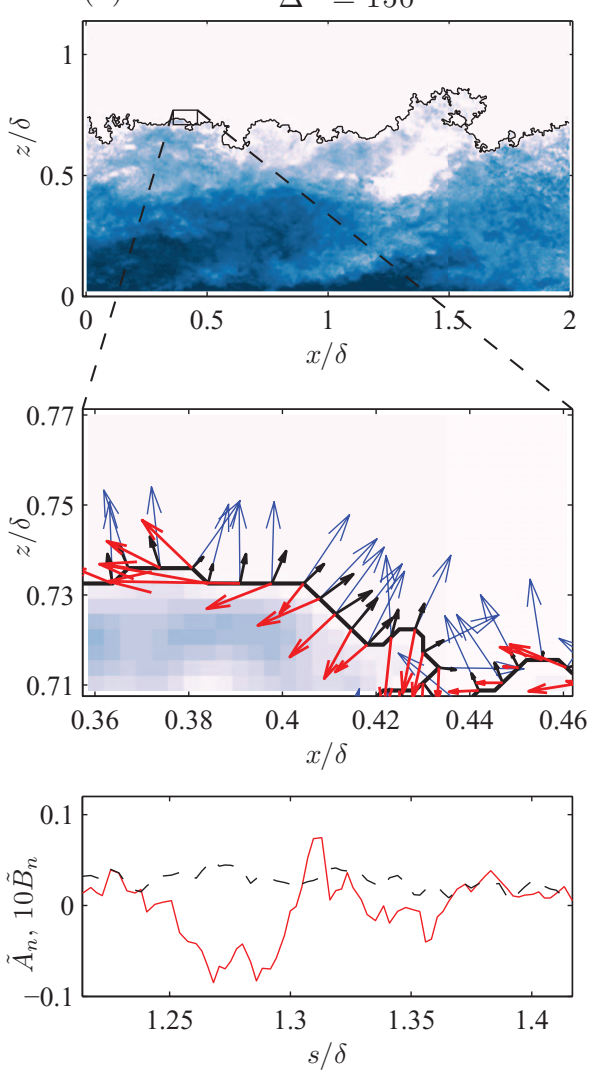

(b)
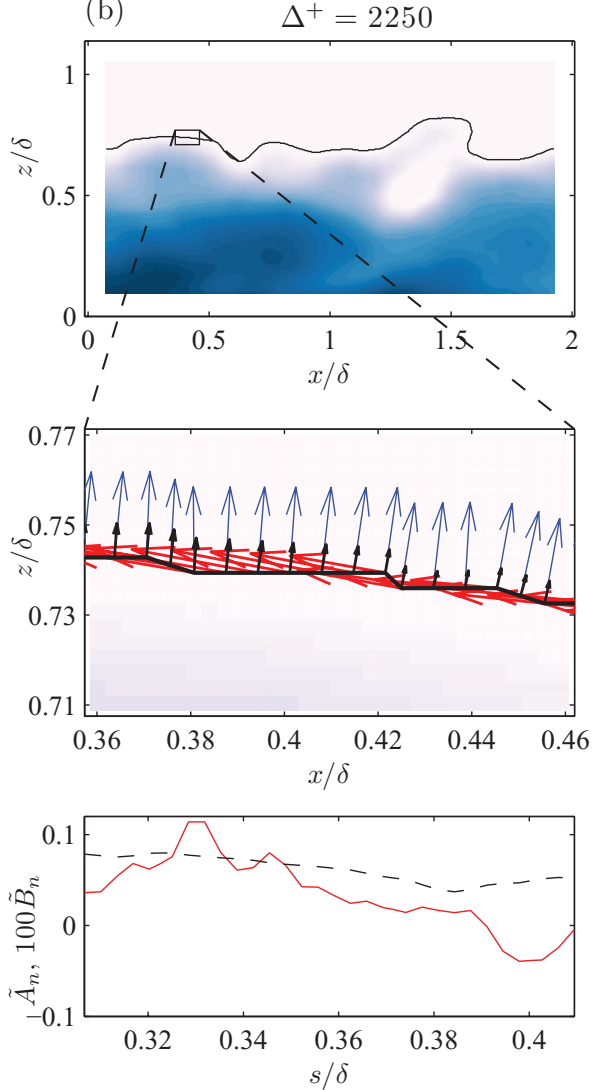

FIG. 8. Components of LES fluxes along the interfaces. (a) Filter size $\Delta^{+}=150$. (b) Filter size $\Delta^{+}=2250$. Top panel: Filtered kinetic energy field along with the interface at a threshold value of $0.1 \%$, with contours of kinetic energy using the same contour colours as shown in Figure 2. Middle panel: Portion of the interface zoomed in from the top panel along with vectors (skipping every other vector); normal to the interface $(\overline{\mathbf{n}})$, blue arrows; the LES advection flux vector $(\tilde{\mathbf{A}}$, with $\left.\tilde{A}_{j} \equiv(1 / 2)\left(\widetilde{u_{j} u_{i} u_{i}}-\tilde{u}_{j} \widetilde{u_{i} u_{i}}\right)\right)$, red arrows; and the LES viscous flux vector $\left(\tilde{\mathbf{B}}\right.$, with $\left.\tilde{B}_{j} \equiv-2 v \widetilde{u_{i} S_{i j}}\right)$, short black arrows. Bottom panel: Magnitude of advective (solid lines) and viscous (dashed lines) flux vectors $\left[\mathrm{m}^{3} \mathrm{~s}^{-3}\right]$ normal to the interface $\left(\tilde{A}_{n}, \tilde{B}_{n}\right)$ for the zoomed view in the middle panel. Note that in both the middle and the bottom panels, the viscous flux vectors are multiplied by a factor 10 in (a) and 100 in (b) for visual clarity.
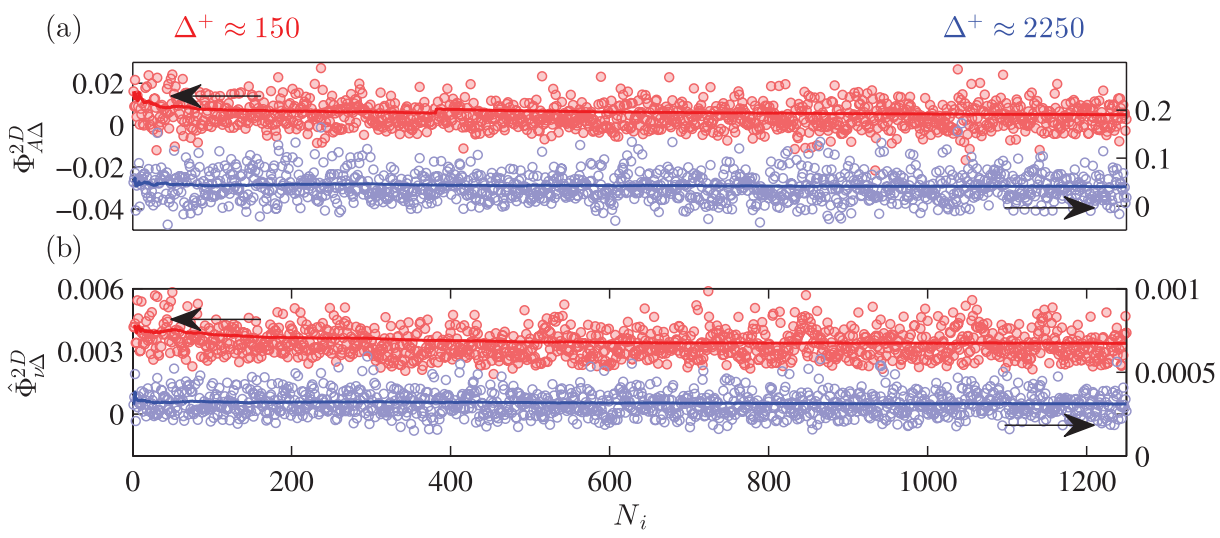

FIG. 9. Variation of (a) $\Phi_{A \Delta}^{2 D}(\Delta)\left[\mathrm{m}^{4} \mathrm{~s}^{-3}\right]$ and (b) $\hat{\Phi}_{v \Delta}^{2 D}(\Delta)\left[\mathrm{m}^{4} \mathrm{~s}^{-3}\right]$ across the 1250 filtered vector fields at $\Delta^{+} \approx 150$ (filled symbols - left ordinate) and $\Delta^{+} \approx 2250$ (empty symbols - right ordinate), solid lines (-) show the convergence of the corresponding mean values with increasing number of images $N_{i}$. 


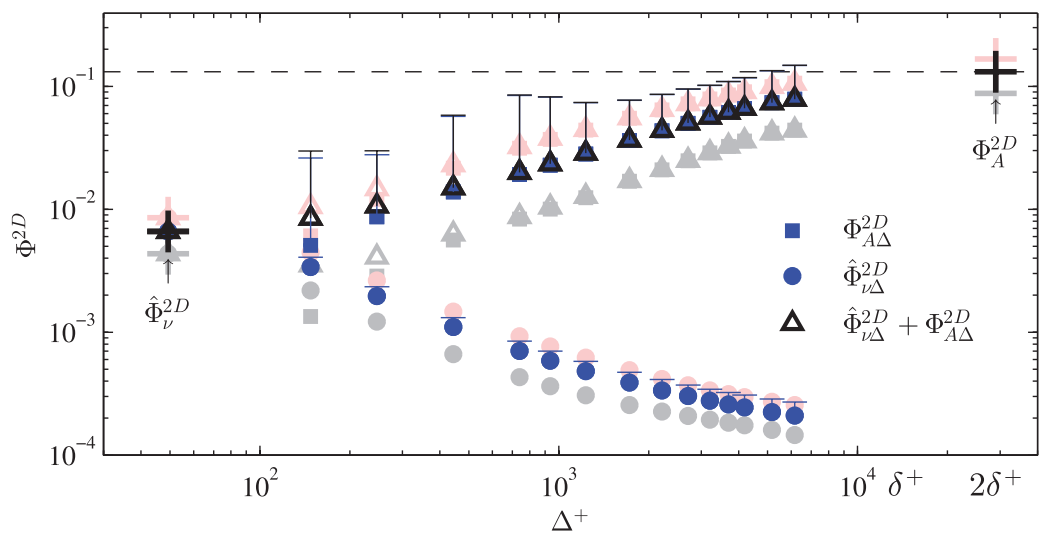

FIG. 10. Comparison of unfiltered, RANS, and LES fluxes $\left[\mathrm{m}^{4} \mathrm{~s}^{-3}\right]$ for the data set at $R e_{\tau}=14500$ (in dark symbols). $\hat{\Phi}_{v}^{2 D}$ and $\Phi_{A}^{2 D}$ are indicated in the figure (+ symbols); the dashed (black) line is $\Phi_{A}^{2 D}$ (independent of scale because it is RANS); $\Phi_{A \Delta}^{2 D}, \boldsymbol{\square} ; \hat{\Phi}_{v \Delta}^{2 D}, \bullet ;$ and $\Phi_{A \Delta}^{2 D}+\hat{\Phi}_{v \Delta}^{2 D}, \Delta$. The "error" bars on the symbols represent one standard deviation, and are plotted on only the top-side. Note that we also show the case of two other threshold levels for detecting the interface, $c_{0}=0.5 \times 10^{-3}$ and $1.5 \times 10^{-3}$ (where $K_{0}=c_{0} \frac{1}{2} U_{\infty}^{2}$ ) in light (grey and rose) symbols. The values for $c_{0}=0.5 \times 10^{-3}$ are the lowest of the three thresholds.

available images. Also, one standard deviation is presented as "error" bars on one side of the symbols. As expected, at the smallest scale, the filtered viscous flux $\hat{\Phi}_{v \Delta}^{2 D}$ coincides with the unfiltered case at the measurement resolution, while $\Phi_{A \Delta}^{2 D}$ is zero since no subgrid-scale fluctuations occur when filtering at the measurement resolution. With increasing filter size $\Delta$, the viscous resolved flux $\hat{\Phi}_{v \Delta}^{2 D}$ decreases while $\Phi_{A \Delta}^{2 D}$ increases. At the largest filter size the latter approaches the purely advective flux in the RANS formulation. Furthermore, all the fluxes are positive and the analysis and the experimental results concomitantly show that the boundary of the turbulent region is growing outward into the non-turbulent region, at all scales in the various representations (unfiltered, filtered, and RANS).

Ideally, as mentioned in Sec. IV B, we would have expected the fluxes from the unfiltered case, RANS, and total multi-scale LES filtered cases to be about the same, independent of the scale $\Delta$. Specifically, at large $\Delta$, the combined multi-scale (LES) flux should have been the same as the advective RANS flux, which it does seem to approach at large scales. The slight difference is attributed to the largest filter size that could be used, which is about $0.5 \delta$, and the trends in the graph show that a larger filter (with an even larger field) might make the total LES fluxes even closer to the RANS value.

On the other end of the scale, for small $\Delta$, even though the viscous (unfiltered) flux $\hat{\Phi}_{v}^{2 D}$ matches the viscous resolved (LES) flux $\Phi_{v \Delta}^{2 D}$, their magnitudes are significantly below those of the RANS case. This is due to several reasons. First, $\hat{\Phi}_{v}^{2 D}$ is the $2 \mathrm{D}$ surrogate of the $3 \mathrm{D} \Phi_{v}$ value. Note that even though $\Phi_{A}^{2 D}$ is also $2 \mathrm{D}$, the difference with $3 \mathrm{D}$ is expected to be zero because the RANS interface is $2 \mathrm{D}$ in the presently considered flow due to the 2D nature of the mean boundary layer flow (specifically, fluxes involving the $x_{2}$ direction vanish). However, at the smallest scales in instantaneous fields, 3D effects are important. Statistically speaking, small-scale isotropy could be invoked to deduce the 3D statistical values from those in 2D. As shown in Appendix C, when assuming local isotropy for the small scales, one can show that 3D fluxes should be about twice the 2D fluxes. Even though this would make $\hat{\Phi}_{v}^{2 D}$ closer to the RANS flux, it is still almost an order of magnitude smaller. Second, the experimental velocity field is obtained at $\Delta x^{+} \approx 50$, which is about 4-10 times larger than the Kolmogorov length scales for $z$-locations between the outer to mean interface location. It is not unusual for PIV to under-predict quantities such as dissipation by an order of magnitude for such $\Delta x / \eta$ values. ${ }^{48,49}$ As cautioned before, we believe lack of spatial resolution may explain the low value of $\hat{\Phi}_{v}$ compared to the RANS-level flux. We also note that in calculating $\Phi_{A}^{2 D}$, the pressure-velocity correlation term could not be included. An estimate of the neglected term $\int(1 / \rho) \overline{u_{3}^{\prime} p^{\prime}} \bar{n}_{3} d s$ (assuming that $\int(1 / \rho) \overline{u_{1}^{\prime} p^{\prime}} \bar{n}_{1} d s$ is negligible due to small $\bar{n}_{1}$ 
compared to $\bar{n}_{3}$ ) made from the DNS kinetic energy balance for a turbulent boundary layer at $\operatorname{Re}_{\tau}$ $=1272$ by Schlatter and Örlü ${ }^{50}$ is $\approx 10 \%$ of the calculated $\Phi_{A}^{2 D}$ with an opposite sign. ${ }^{51}$

The effect of the threshold level on the variations in the calculated fluxes are exhibited in Figure 10 by plotting the un-averaged, filtered and RANS fluxes for $c_{0}=0.5 \times 10^{-3}$ and $1.5 \times$ $10^{-3}$ (recall that $K_{0}=c_{0} \frac{1}{2} U_{\infty}^{2}$ ) in lighter symbols. Even though there is slight shift in the measured distributions, the overall trend is the same.

\section{E. Scaling of fluxes and the entrainment velocity}

To understand the scaling of the fluxes the calculations similar to Sec. V D are repeated for the database at $R e_{\tau}=7870$ (using the same threshold value of $0.1 \%$ ). The PIV velocity fields of Adrian, Meinhart, and Tomkins ${ }^{29}$ at $R e_{\tau}=2790$ are also used to calculate the fluxes (even though there are only 50 realisations available and we have to employ a threshold value of $0.15 \%$ ). Results for the measured fluxes shown in Sec. V D were in dimensional units, i.e., they were not normalised. In order to compare results at different $R e_{\tau}$, it useful to define fluxes per unit interface length, accordingly,

$$
\hat{\Psi}_{v}^{2 D} \equiv \frac{1}{l_{s}} \hat{\Phi}_{v}^{2 D},
$$

where $l_{s}$ is the total interface length of the individual fields, i.e., $\hat{\Psi}_{v}^{2 D}$ is the viscous flux per unit length. It is made non-dimensional by $u_{\tau}$, i.e., $\hat{\Psi}_{v}^{2 D+}=\hat{\Psi}_{v}^{2 D} / u_{\tau}^{3}$, and a similar definition is also used for the advective flux. Normalised fluxes for $R e_{\tau}=14500,7870$, and 2790 are plotted in Figures 11(a)-11(c) with the abscissa normalised by inner, outer, and Taylor microscale (at the mean interface location of $\approx 0.67 \delta$ ), respectively. A reasonable collapse of $\hat{\Psi}_{v}^{2 D+}$ at the ordinate for the three $R e_{\tau}$ data (even though $\Phi$ differ by more than an order of magnitude between the different Reynolds numbers) seems to suggest that the flux per unit length does scale with $u_{\tau}^{3}$. This might not be surprising, considering the fact that $u^{\prime}$ (the turbulent velocity fluctuations) scale almost linearly with $u_{\tau}$ even as far from the wall as the interface location. The role of local fluctuating velocity in the propagation of the interface has also been observed by Holzner et al. ${ }^{52}$ in their experiments and DNS with oscillating-grids.

Note that the inner and outer normalisation (see Figures 11(a) and 11(b), respectively) tend to collapse the small and the large $\Delta \mathrm{s}$ differently. The viscous scaling is less satisfactory at all $\Delta$, whereas a better collapse of large scales with outer scaling is simply because large eddies scale with the boundary layer thickness $\delta$. On the other hand, $\lambda_{T}$ seems to collapse the data well for the lower/mid range of $\Delta$. This is likely because length scales which dictate the entrainment at small scales are of the order of Taylor microscale (e.g., da Silva and Taveira ${ }^{27}$ have shown that the radius of the vortices which reside along the interface are comparable to the large vorticity structures and are of the order of Taylor microscale in shear-flows). The significance of $\lambda_{T}$ at smaller scales and that of $\delta$ at largest scales is also exemplified in the "mass-entrainment spectrum" presented by Chauhan et al. ${ }^{25}$ which characterizes the length scales at which the local and the total mass are entrained into the boundary layer. As an aside, we note that the Kolmogorov length scale $\eta^{+}$is relatively invariant with $R e_{\tau}$ in the outer region of the boundary layer, and the variation in Taylor micro-scale $\lambda_{T}^{+}$is moderate. ${ }^{53}$

Finally, some observations regarding entrainment velocities are made. Recalling the requirement (from the fact that total flux is constant at any scale, see Eqs. (28) and (D7)), that $v_{s}^{\Delta} S(\Delta)$ is a constant, if the surface area $S(\Delta)$ follows fractal like scaling $S(\Delta) \sim \Delta^{2-D_{f}}$ with a fractal dimension of approximately $D_{f}=7 / 3$ (e.g., see, Ref. 19), then $S(\Delta) \sim \Delta^{-1 / 3}$. This should hold within a range of scales between an inner and outer cutoff scale. This leads to the conclusion that the average entrainment velocity at scale $\Delta$ follows,

$$
v_{s}^{\Delta}(\Delta) \sim \Delta^{1 / 3},
$$

consistent with inertial-range Kolmogorov scaling of transport velocities. We cannot, however, verify this prediction since even though the surface area scales as $S(\Delta) \sim \Delta^{-1 / 3}$, the total flux $\left(\Phi_{A \Delta}+\Phi_{v \Delta}\right)$ is not observed to be independent of scale (see Figure 10) due to possibly scale-dependent effects from $3 \mathrm{D}$ to $2 \mathrm{D}$ corrections and resolution issues at the smallest scales. Due to $2 \mathrm{D}$ measurement 

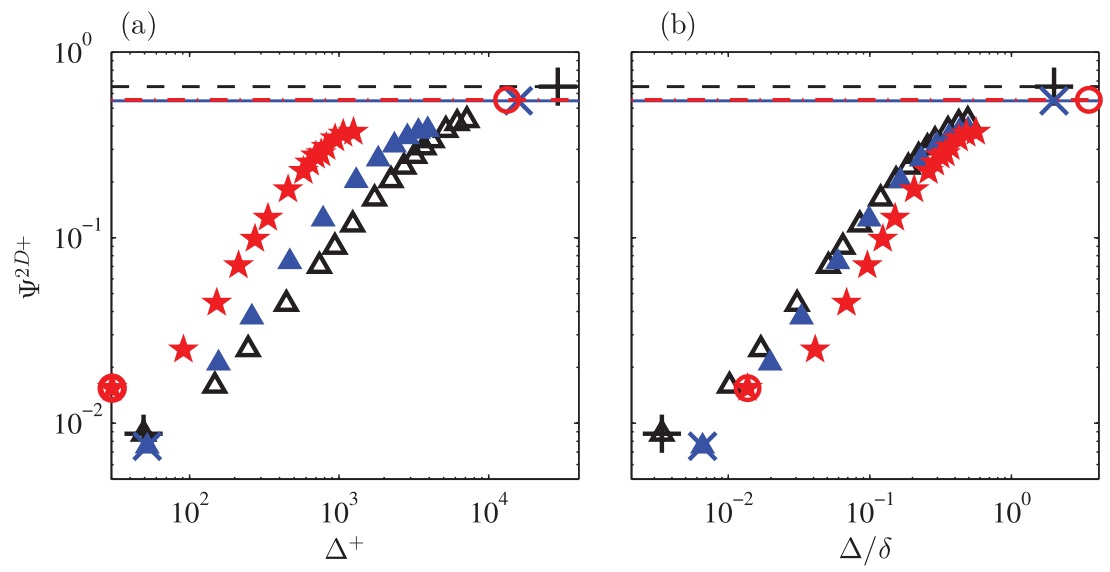

(c)

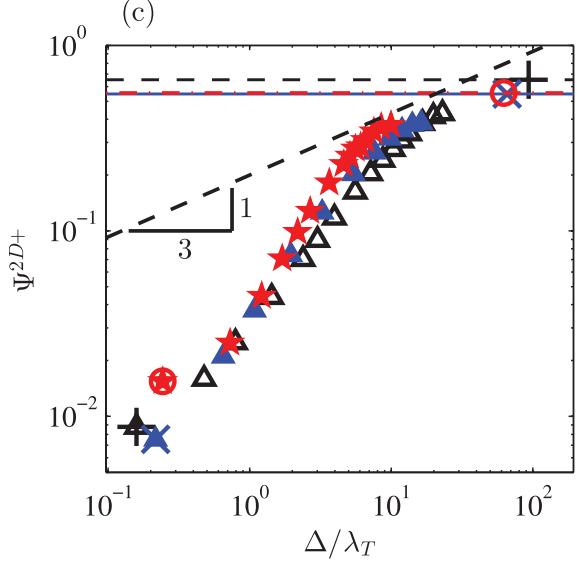

FIG. 11. The normalised fluxes per unit interface length $\Psi^{2 D} \equiv\left(1 / l_{s}\right) \Phi^{2 D},\left(\Psi_{A \Delta}^{2 D+}+\hat{\Psi}_{v \Delta}^{2 D+}\right)$ at $R e_{\tau}=14500$ (black $\left.\triangle\right), R e_{\tau}$ $=7870$ (blue $\mathbf{\Lambda}$ ), and $R e_{\tau}=2790$ of Adrian, Meinhart, and Tomkins (red $\star$ ). Normalisation of abscissa with (a) viscous units $\Delta^{+}=\Delta /\left(v / u_{\tau}\right)$, (b) outer units $\Delta / \delta$, and (c) Taylor microscale $\Delta / \lambda_{T}$. (a), (b), and (c) also show $\hat{\Psi}_{v}^{2 D+}$ and $\Psi_{A}^{2 D+}$ denoted by ,$+ \times$, and $\circ$ for $R e_{\tau}=14500,7870$, and 2790, respectively. The horizontal dashed, solid, and dashed-dotted lines correspond to $\Psi_{A}^{2 D+}$ for $R e_{\tau}=14500,7870$, and 2790, respectively. Note that "entrainment velocity" is $v_{s}^{\Delta 2 D}=\left(\Psi_{v \Delta}^{2 D}+\Psi_{A \Delta}^{2 D}\right) / K_{0}$, and therefore the figures also present scaling of the $v_{s}^{\Delta 2 D}$. Scaling of $v_{s}^{\Delta 2 D} \sim \Delta^{1 / 3}$ is observed when the fluxes at all scales are equal.

planes, we are measuring the length and not the surface area, and the entrainment velocity is in a 2D plane. For the sake of present discussion, let us denote the 2D surrogate as $v_{s}^{\Delta 2 D}$. As it turns out, the scaling in 3D holds for the 2D case also, $v_{s}^{\Delta 2 D} \sim \Delta^{1 / 3}$ because the total length $l_{s}$ at any scale $\Delta$ is now $l_{s}(\Delta) \sim \Delta^{1-d}$, with $d=4 / 3$ due to the additivity rules of fractal dimensions in planar intersections. Furthermore, $v_{s}^{\Delta}=\left(\Psi_{v \Delta}+\Psi_{A \Delta}\right) / K_{0}$, and similarly $v_{s}^{\Delta 2 D}=\left(\Psi_{v \Delta}^{2 D}+\Psi_{A \Delta}^{2 D}\right) / K_{0}$. To illustrate such a scaling, $v_{s}^{\Delta 2 D} \sim \Delta^{1 / 3}$ is indicated in Figure 11(c) with a line of slope 1/3. Not surprisingly, the data show a larger slope at small $\Delta$, because the fluxes are underestimated. Some indication that the slope of the data may tend towards $1 / 3$ can be seen at increasing $\Delta$.

\section{SUMMARY AND CONCLUSIONS}

Properties of various transport mechanisms across the turbulent/non-turbulent interface are examined via a multi-scale analysis of the kinetic energy equation and experimental data obtained in high Reynolds number turbulent boundary layers. Operationally, the TNTI is identified with a kinetic energy $(K)$ threshold applied to the experimental PIV data. Our analysis shows that it is the viscous stresses that transport $K$ across the interface (referred to here as the "nibbling" process), whereas if the same analysis is carried out for an "averaged flow" (ensemble - or Reynolds averaged formulation), the transport is dominated by the advective fluxes (considered in this paper as representative of 
the "engulfment" process) with negligible viscous contributions. In between, a multi-scale analysis by filtering the fields at particular length scales shows that except at the smallest and largest scales, both viscous (nibbling) and advective (engulfing) processes are active. Furthermore, the analysis suggests that independent of the scale, the total flux should be approximately the same. Consequently, the results are consistent with "engulfment" and "nibbling" being viewed as describing the same entrainment process, however, at the largest and smallest scales, respectively. At in-between length scales both advective and nibbling contribute to the overall entrainment. We stress again that the equivalency between the concept of "large-scale engulfment" and the more precisely defined "advective flux" at various scales that we have assumed here may not correspond precisely to prior interpretations or definitions of "large-scale engulfment" and this should be kept in mind.

Two-dimensional experimental data from PIV in turbulent boundary layers at $R e_{\tau}$ of 14500 and 7870 (along with a relatively smaller data-set of Adrian, Meinhart, and Tomkins ${ }^{29}$ at $R e_{\tau}=$ 2790) are analysed to understand the specific nature of the viscous and advective fluxes operative at the interface. At the smallest scale (describing the nibbling process), the viscous fluxes drive the growth of the boundary layer by the work done by the viscous stresses. This is confirmed by the strong negative correlation of the normal/tangential velocities and their gradients along the interface. Qualitatively the process of entrainment by viscous fluxes wherein the viscous flux vector is aligned with the local interface normal remains the same at increasing scales even though the flux magnitudes decrease rapidly as the filtering scale is increased. Advective fluxes on the other hand drive entrainment primarily due to their large magnitudes, rather than any particular alignment with the local interface. At large scales, the interface flattens and the local normals to the interface become less random, making the alignment between the advective flux vector and local normal slightly higher. At the largest scales, where the entire entrainment is driven by advective fluxes (describing the engulfment process), two specific contributions occur equally: the mean flow and shear-stress interaction, and the advection of $K$ due to turbulent fluctuations. The former is related entirely to the mean flow (due to the relation given by Reynolds, ${ }^{21}$ between shear-stress and mean tangential velocity jump) and has been the one studied mostly in the literature. However, it is shown that for entrainment the advection by turbulent fluctuations is also equally important. The multi-scale flux calculations based on the LES filtering formalism show that with increasing scale the advective flux contribution becomes dominant over the (resolved) viscous contribution. As a result, for most intermediate scales (except for the smallest), advective fluxes prevail over nibbling.

While the present PIV data provide us with sufficient information at high Reynolds numbers to describe the overall trends of each of the processes correctly, lack of spatial resolution deep into the viscous range yields viscous nibbling fluxes that are smaller than the advective ones that operate at the large scales. Nonetheless, the transport equation clearly shows that viscous nibbling must rise sufficiently to balance the overall entrainment flux that determines the growth of the turbulent boundary layer.

Viscous and advective fluxes are analysed at multi-scales for three different Reynolds numbers from two different laboratories, and collapse well when the fluxes are converted to fluxes per unit interface length and normalised with $u_{\tau}^{3}$. This shows that $u_{\tau}$ or the local fluctuating velocity (since the local fluctuating velocity in the outer region scales with $u_{\tau}$ ) in the laboratory frame (rather than attached to the interface) is the appropriate velocity scale (in general agreement with Holzner et al. ${ }^{52}$ ). When length-scales are normalized with the Taylor scale, reasonable collapse is observed in the small/mid length scales, highlighting the importance of the Taylor scale. This may be expected since we recognize that the definition of the nibbling flux (Eq. (7)) involves the product of the viscous shear stress, a small-scale quantity, with the local velocity, a large-scale one. On the other hand, fluxes at larger $\Delta$ seem to scale with $\delta$ similar to the largest eddies in the outer region of the boundary layer. Furthermore, scaling of the entrainment velocity $v_{s}^{\Delta} \sim \Delta^{1 / 3}$ derived by considering a multiscale (fractal) interface and constant flux across scales could not be verified due to under-estimation of viscous fluxes at smaller $\Delta$, however, approaches such a scaling at larger scales.

These results provide evidence at higher Reynolds numbers that interfaces in turbulence are accompanied by transport processes that depend on scale. The results suggest that the overall rate of entrainment is determined by the large-scales but that the actual entrainment occurs physically at the small diffusive scales along the interface. Nevertheless, lack of sufficient spatial resolution at 
the smallest scales prevented us from capturing the full magnitude of the viscous fluxes and hence the data do not yet fully prove whether once viscous fluxes are fully resolved they match the ones from the large scales. Nevertheless, the theoretical analysis of the energy budget and overall growth of the interface indicates that this must be the case.

\section{ACKNOWLEDGMENTS}

The authors wish to thank Dr. Kapil Chauhan and Dr. Daniel Chung for fruitful discussions, and the Australian Research Council for the financial support of this work. The visit of C.M. to the University of Melbourne was supported by the Australian-American Fulbright Commission Senior Scholar Fellowship and a University of Melbourne MERIT Visiting Fellowship. C.M. also acknowledges partial funding from the NSF (Grant No. CBET -1033942).

\section{APPENDIX A: EFFECTS FROM IRROTATIONAL KINETIC ENERGY WITHIN THE CONTROL VOLUME $v_{o}$}

Here we consider terms that appear in (8). We begin with the closed-surface integral $\iint_{S_{o}}\left(K+\frac{p}{\rho}\right)\left(u_{i}-U_{i}\right) n_{i} d S$ containing the inertial terms. If we now assume that the flow outside the interface is mostly irrotational, we may apply the unsteady Bernoulli equation between any point on the boundary and the far-field, $K+p / \rho=p_{\infty} / \rho-\partial \phi / \partial t$ (where $\phi$ is the velocity potential), and obtain

$$
\iint_{S_{o}}\left(K+\frac{p}{\rho}\right)\left(u_{i}-U_{i}\right) n_{i} d S \approx-\iint_{S_{o}}\left(\frac{\partial \phi}{\partial t}\right)\left(u_{i}-U_{i}\right) n_{i} d S
$$

since $\iint_{S_{o}}\left(p_{\infty} / \rho\right)\left(u_{i}-U_{i}\right) n_{i} d S=0$ from mass conservation $\left(\iint_{S_{o}}\left(u_{i}-U_{i}\right) n_{i} d S=0\right)$. The closed surface integral on the right hand side of (A1) can be written (recalling that $\mathbf{n}$ points inwards into $\left.V_{o}\right)$ as

$$
-\iint_{S_{o}}\left(\frac{\partial \phi}{\partial t}\right)\left(u_{i}-U_{i}\right) n_{i} d S=\iiint_{V_{o}} \frac{\partial}{\partial x_{i}}\left(\frac{\partial \phi}{\partial t} \frac{\partial \phi}{\partial x_{i}}\right) d V=\iiint_{V_{o}}\left(\frac{\partial}{\partial t} \frac{\partial \phi}{\partial x_{i}}\right)\left(\frac{\partial \phi}{\partial x_{i}}\right) d V,
$$

where we have assumed that $u_{i}-U_{i}=\partial \phi / \partial x_{i}$ and used $\nabla^{2} \phi=0$. This leads to

$$
-\iint_{S_{o}}\left(\frac{\partial \phi}{\partial t}\right)\left(u_{i}-U_{i}\right) n_{i} d S=\iiint_{V_{o}} \frac{\partial}{\partial x_{i}}\left(\frac{\partial \phi}{\partial t} \frac{\partial \phi}{\partial x_{i}}\right) d V=\frac{d \mathcal{K}_{V_{o}}^{\mathrm{pot}}}{d t},
$$

where $\mathcal{K}_{V_{o}}^{\text {pot }}=\iiint_{V_{o}}(1 / 2)\left(\partial \phi / \partial x_{i}\right)^{2} d V$ is the kinetic energy in the potential-flow portion of the fluctuations outside of the interface.

The other term in (8) that needs to be considered is the surface integration $\iint_{S_{o}} K U_{i} n_{i} d S=$ $K_{0} \iint_{S_{\text {int }}} U_{1} n_{1} d S+U_{1} \iint_{S_{L}} K n_{1} d S+\iint_{S_{U}} K U_{1} n_{1} d S$. The integral over the interface $S_{\text {int }}$ was already considered in the main text, while the integral over $S_{U}$ vanishes since $n_{1}=0$ there. Along $S_{L}$, the inward pointing normal has $n_{1}=1$. Along this surface $K<K_{0}$ and is likely small. Consistent with the previous reasoning we assume the fluctuations at most contain irrotational contributions. Let $\left\langle K^{\text {pot }}\right\rangle_{L}$ be the average value of these fluctuations along $S_{L}$. Typically one expects $\left\langle K^{\text {pot }}\right\rangle_{L} \ll K_{0}$, especially if $K^{\text {pot }}$ decays rapidly away from the interface. Combined with the arguments in the main text leading to (10), the surface integral can be written as

$$
\iint_{S_{o}} K U_{i} n_{i} d S=-U_{1} K_{0} \frac{d \delta}{d x} A_{x y}+U_{1}\left\langle K^{\mathrm{pot}}\right\rangle_{L} \frac{d \delta}{d x} A_{x y}
$$

Finally, if one includes explicit consideration of the irrotational portion of the kinetic energy in $V_{o}$, (10) can instead be written according to

$$
\frac{d}{d t}\left(\mathcal{K}_{V_{o}}-\mathcal{K}_{V_{o}}^{\mathrm{pot}}\right)+\left(K_{0}-\left\langle K^{\mathrm{pot}}\right\rangle_{L}\right) U_{1} \frac{d \delta}{d x} A_{x y}=\Phi_{v}-D
$$


In other words, it can be seen that the flux $\Phi_{v}$ affects the portion of the outer kinetic energy that is assumed to be rotational. It will have no effect on the irrotational portions, which instead can be affected by pressure work at the interface. We note that in the analysis of the experimental database we always consider a frame of reference moving with the free-stream velocity (i.e., $U_{1}=0$ ), and the second term in (A5) is identically zero.

At this point, it might be interesting to have an estimate of the term $d / d t\left(\mathcal{K}_{V_{0}}^{\mathrm{pot}}\right)$. To this end, we employ the expression of Phillips ${ }^{2}$ for the decay of kinetic energy in the potential region due to random fluctuations at the interface, according to which the kinetic energy $\sim h^{-4}$, where $h$ is the distance from the interface. It is experimentally validated by Bradshaw ${ }^{54}$ for the case of a turbulent boundary layer and the expression seems to hold even inside the intermittent region of the boundary layer. Therefore, we consider the (potential) kinetic energy of the irrotational fluid in the control volume $V_{0}$, which is assumed to vary like $K^{\text {pot }}=K_{0}(z / \delta)^{-4}$, where $z$ is the wall-normal distance. Note that at $z=\delta$, we have the usual $K^{\text {pot }}=K_{0}$. Hence, the total potential kinetic energy in the control volume $V_{0}$ per unit spanwise width (with $\delta=\delta_{0}+x c$, where the constant $c=d \delta / d x$ ) is

$$
\begin{aligned}
\mathcal{K}_{V_{0}}^{\mathrm{pot}}=K_{0} \int_{x=0}^{L_{x}} \int_{z=\delta(x)}^{h}\left(\frac{z}{\delta}\right)^{-4} \mathrm{~d} z \mathrm{~d} x & =\frac{K_{0}}{3} \int_{x=0}^{L_{x}}\left(\delta-\frac{\delta^{4}}{h^{3}}\right) \mathrm{d} x \\
& =\frac{K_{0}}{3}\left[\delta_{0} L_{x}+\frac{c L_{x}^{2}}{2}-\frac{1}{5 h^{3} c}\left\{\left(\delta_{0}+c L_{x}\right)^{5}-\delta_{0}^{5}\right\}\right],
\end{aligned}
$$

where, $L_{x}$ is the length of the streamwise domain under consideration, and we have taken the control volume to extend up to a constant height $h$ in the $z$-direction. Now, $\delta_{0}$ is a function of time (such that in a frame of reference moving with the free-stream velocity the boundary layer moves outwards), and $d \delta_{0} / d t=U_{\infty} d \delta /\left.d x\right|_{\delta_{0}}=U_{\infty} c$. This suggests that (with $K_{0}=c_{0}\left(\frac{1}{2} U_{\infty}^{2}\right), c_{0}=10^{-3}$ ),

$$
\Psi^{2 D \mathrm{pot}+} \equiv \frac{1}{L_{x} u_{\tau}^{3}} \frac{d \mathcal{K}_{V_{0}}^{\mathrm{pot}}}{d t}=\frac{c_{0} U_{\infty}^{+3} c}{6}\left[1-\left(\frac{c L_{x}}{h}\right)^{3}\left\{\left(\frac{\delta_{0}}{c L_{x}}+1\right)^{4}-\left(\frac{\delta_{0}}{c L_{x}}\right)^{4}\right\}\right] .
$$

In the present experiments for the case of $R e_{\tau}=14500$, we have $c=0.012$ and $U_{\infty}^{+}=31.75$. An upper bound of $\Psi^{2 D \text { pot }+}$ results when we extend the volume to $\infty$, or in the limit $h / \Delta \rightarrow \infty$, and this results in $\Psi^{2 D \text { pot }+} \approx 6.4 \times 10^{-2}$. However, for a realistic and finite $h$, say $h=2 \delta, \Psi^{2 D \text { pot }+} \approx 3.2$ $\times 10^{-2}$. These values of $\Psi^{2 D \text { pot+ }}$ should be compared to the distribution of $\Psi^{2 D+}$ in Figure 11, and shows that maximum of $\Psi^{2 D \text { pot }+}$ is about $5 \%-10 \%$ of the RANS case $\Psi_{A}^{2 D+}$. Note that, we expect $\Psi^{2 D \text { pot }+}$ to be relatively invariant with the filter size $\Delta$, since the average RANS interface and the un-averaged interface are nominally at the same $z$-location.

\section{APPENDIX B: INTERFACE FOR MULTI-SCALE ANALYSIS}

The interface is defined with the same threshold of $K=10^{-3}\left(\frac{1}{2} U_{\infty}^{2}\right)$ for each formalism, based on unfiltered fields, RANS, and filtered (LES) fields. Specifically, $\tilde{K} \equiv(1 / 2)\left(\tilde{u}^{2}+\tilde{w}^{2}\right)$ for the LES case and $\bar{K} \equiv(1 / 2)\left(\bar{u}^{2}+\bar{w}^{2}\right)$ for the RANS case, in a co-ordinate system which moves with the free stream velocity (i.e., $\mathbf{U}=\mathbf{0}$ ). Due to the nonlinear nature of the definition of $K$ it is not obvious if using the same threshold would yield an interface which (at least visually) could be considered as the same interface expressed at various resolutions. In this appendix, we provide empirical evidence that indeed the basic definition and the same threshold enable us to roughly identify the same interface at various resolutions.

By taking a single field as an example, Figure 12 shows various ways in which $K$ could be defined. It shows the original interface along with three definitions of $\tilde{K}$ at $\Delta^{+} \approx 950$ : (i) $\widetilde{K_{b i n}}$ obtained by filtering the binary (T/NT) image (with the turbulent region assigned a value unity and zero otherwise, and obtaining an interface at the value of 0.5 after filtering), (ii) calculating $K$ and then filtering, i.e., $(1 / 2)\left(\widetilde{u^{2}+w^{2}}\right)$, and (iii) first filtering and then calculating $K$, i.e., $(1 / 2)\left(\tilde{u}^{2}+\tilde{w}^{2}\right)$. Out of the three, the third is the most logical definition which is extendable to any scale, and the one used in the main text. Figure 12 shows that visually they all appear similar and not far from the 
$\Delta^{+} \approx 950$

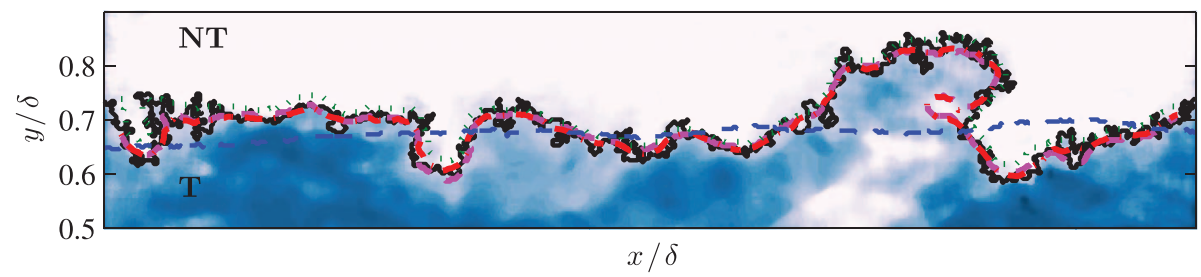

FIG. 12. A snapshot of the velocity field relative to the free-stream at $R e_{\tau}=14500$ from planar PIV. The solid black line indicates the location of the TNTI computed as an iso-kinetic energy surface using a threshold of $K=10^{-3}\left(0.5 U_{\infty}^{2}\right)$. The same TNTI is computed using different filtering techniques to compute $\tilde{K}$ at a scale $\Delta^{+} \approx 950$, where the dotted, dash-dotted, and dashed lines represent $\widetilde{K_{b i n}}$ (for definition see text), $(1 / 2)\left(\widetilde{u^{2}+v^{2}}\right)$ and $(1 / 2)\left(\tilde{u}^{2}+\tilde{v}^{2}\right)$, respectively. TNTI location averaged across 1250 fields for the RANS case is indicated by the dashed (blue) line. Colour contours are the same as presented in Figure 2.

unfiltered interface. With $\tilde{K}$ as the definition, Figure 12 also shows the RANS interface (i.e., first ensemble averaging the fields and then using the threshold of $0.1 \%$ ) in a (blue) dashed line.

\section{APPENDIX C: ON THE ISOTROPY OF VISCOUS FLUXES ALONG THE INTERFACE}

Viscous fluxes engage in enlarging the turbulent region at the smallest scales and it might be interesting to ascertain to what extent these fluxes at the small scales exhibit local isotropy. If local isotropy holds true, then we can use the 2D data to deduce the 3D flux, and help fill the current measurement gap.

To consider the isotropic relation for the viscous flux $\left\langle 2 v S_{i j} u_{j} n_{i}\right\rangle_{s}$ (where \langle\rangle$_{s}$ denotes average along the interface as well as over ensembles), we begin with the general fourth order tensor, which under the assumption of isotropy is given by

$$
\left\langle S_{i j} u_{p} n_{q}\right\rangle_{s}=\alpha \delta_{i j} \delta_{p q}+\beta \delta_{i p} \delta_{j q}+\gamma \delta_{i q} \delta_{p j},
$$

where, $i, j, p, q=1,2,3$, and $\alpha, \beta$, and $\gamma$ are constants to be determined. Conditions of incompressibility $\left(S_{i i}=0\right)$ and the symmetry of viscous stress tensor (or $S_{i j}=S_{j i}$ ) lead to

$$
\left.\begin{array}{l}
\begin{array}{l}
3 \alpha+\beta+\gamma=0 \\
\beta-\gamma=0
\end{array}
\end{array}\right\}
$$

Equation (C2) yields $\alpha=-(2 / 3) \beta$ and $\gamma=\beta$, which when replaced in (C1) furnishes the expressions for the contractions needed for the $3 \mathrm{D}$ and $2 \mathrm{D}$ cases:

$$
\left.\begin{array}{l}
\left\langle 2 v S_{i j} u_{j} n_{i}\right\rangle_{s}=20 v \beta ; \\
\left.\left\langle 2 v S_{i j} u_{j} n_{i}\right\rangle_{s}\right|_{2 D}=\frac{28}{3} v \beta,
\end{array}\right\},
$$

where, for $\left.\left\langle 2 v S_{i j} u_{j} n_{i}\right\rangle_{s}\right|_{2 D}$, we use $i, j=1,3$. This shows that the $2 \mathrm{D}$ expression will under-predict the viscous flux by a factor of $15 / 7$, i.e., about 2 .

The validity of isotropy can be checked with the measured data, for which the following individual expressions are useful:

$$
\left.\begin{array}{r}
\left\langle S_{11} u_{1} n_{1}\right\rangle_{s}=\left\langle S_{22} u_{2} n_{2}\right\rangle_{s}=\left\langle S_{33} u_{3} n_{3}\right\rangle_{s}=\frac{4}{3} \beta ; \\
\left\langle S_{12} u_{1} n_{2}\right\rangle_{s}=\left\langle S_{21} u_{2} n_{1}\right\rangle_{s}=\left\langle S_{31} u_{3} n_{1}\right\rangle_{s}=\cdots=\beta ; \\
\left\langle S_{12} u_{1} n_{1}\right\rangle_{s}=\left\langle S_{21} u_{1} n_{1}\right\rangle_{s}=\left\langle S_{31} u_{1} n_{1}\right\rangle_{s}=\cdots=0 .
\end{array}\right\} .
$$

The mean values of $\left\langle S_{11} u_{1} n_{1}\right\rangle_{s}$ and $\left\langle S_{22} u_{2} n_{2}\right\rangle_{s}$ in $\left[\mathrm{ms}^{-2}\right]$ are -21.4 and -11.4 , whereas those of $\left\langle S_{12} u_{1} n_{2}\right\rangle_{s}$ and $\left\langle S_{21} u_{2} n_{1}\right\rangle_{s}$ are -14.4 and -5.7 . To the degree that the average of the latter two $(-10.1)$ is smaller in magnitude than the average of the first two $(-16.4)$, and with a ratio of 
(a)

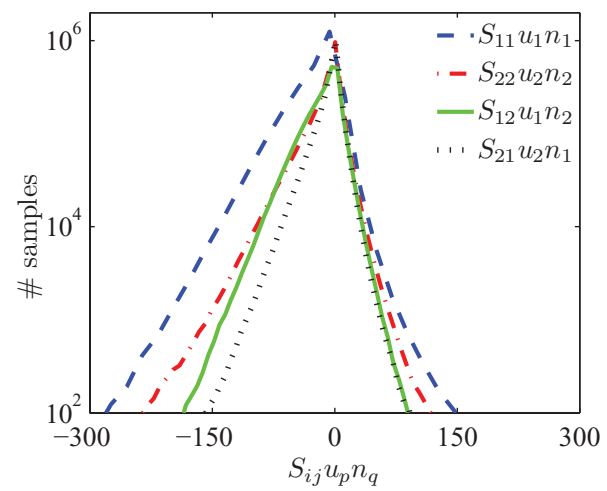

(b)

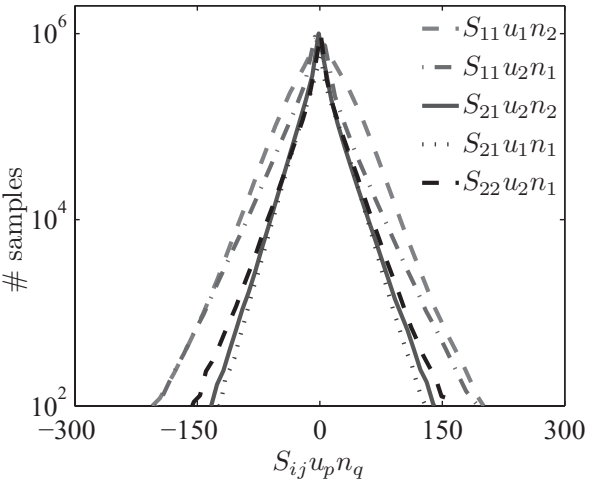

FIG. 13. Density distribution of measured components of the tensor $S_{i j} u_{p} n_{q}\left[\mathrm{~ms}^{-2}\right]$ along the interface for the case of $R e_{\tau}$ $=14500$ across all images. (a) Components for which the mean $\left\langle S_{i j} u_{p} n_{q}\right\rangle_{s} \neq 0$ under isotropy. (b) Components for which the mean $\left\langle S_{i j} u_{p} n_{q}\right\rangle_{s}=0$ under isotropy.

16.4/10.1 of the same order of magnitude as 4/3, the trends agree with small-scale isotropy. The mean values of $\left\langle S_{11} u_{1} n_{2}\right\rangle_{s},\left\langle S_{11} u_{2} n_{1}\right\rangle_{s},\left\langle S_{21} u_{2} n_{2}\right\rangle_{s},\left\langle S_{21} u_{1} n_{1}\right\rangle_{s}$, and $\left\langle S_{22} u_{2} n_{1}\right\rangle_{s}$ are 0.7, -2.6, -0.9, -0.2 , and 0.2 , respectively. These should be equal to zero according to isotropy, and the fact that they are at most about $10 \%$ of the other terms is consistent with isotropy. However, clearly there are significant deviations (factors $\sim 2$ ) from predictions of isotropy.

To also obtain an impression of the distribution functions of these quantities, Figure 13 shows histograms of the components of $S_{i j} u_{p} n_{q}$ calculated from the 2D database at $R e_{\tau}=14500$. The distributions shown in Figure 13(a) compared to those in (b) show that non-zero values arise from non-symmetric distributions in (a) versus symmetric ones in (b).

It is known from Sec. V A that $\mathbf{B}$ is predominantly in the direction of $\mathbf{n}$, and in our case the interface is preferentially growing in an upward direction (i.e., a preferential rather than a uniform density distribution of the angles between $\mathbf{n}$ and $\mathbf{i}$ ). This is a probable cause of the lack of strong isotropy of the viscous fluxes in the present experiments. In the case of "internal interfaces" 14 where there is no overall preferred growth direction of the interface, isotropy of viscous fluxes might be better satisfied.

\section{APPENDIX D: ENTRAINMENT VELOCITY AT MULTI-SCALES}

The purpose here is to carry out an analysis similar to Secs. III, IV A, and IV B; however, with an outer control volume which is deforming with its lower bounding surface attached to the TNTI rather than being fixed. As usual, based on the Reynolds transport theorem, all final physical variables and net fluxes will be equivalent. However, in a formulation with a deforming control surface attached to the TNTI it is more natural to define an entrainment velocity.

Starting with the transport equation for the defect kinetic energy, integrating over the deforming outer control volume, $V_{d o}(\mathbf{x}, t)$, and denoting $\mathbf{u}_{I}$ as the local velocity of the surface or the interface we obtain,

$$
\frac{d \mathcal{K}_{V_{d o}}}{d t}=\iint_{S(\mathbf{x}, t)} K\left(u_{i}-u_{I i}\right) n_{i} \mathrm{~d} S+\iint_{S(\mathbf{x}, t)}\left(\left(u_{j}-U_{j}\right) \frac{p}{\rho} \delta_{i j}-\left(u_{j}-U_{j}\right) \tau_{j i}^{v}\right) n_{i} \mathrm{~d} S-D
$$

where $\mathcal{K}_{V_{d o}} \equiv \iiint_{V_{d o}(\mathbf{x}, t)} K \mathrm{~d} V$ and $D$ is the dissipation inside $V_{d o}(\mathbf{x}, t)$. Now, the local entrainment velocity is the difference of the interface velocity and the material velocity $\mathbf{v}=\mathbf{u}_{I}-\mathbf{u}$ (see, e.g., Ref. 47). The kinetic energy inside $V_{d o}$ is approximately zero at all times, therefore, even though $V_{d o}$ changes with time, $d \mathcal{K}_{V_{d o}} / d t \approx 0$. Moreover, following Sec. III, i.e., using the Bernoulli equation 
$K_{0}+p / \rho=p_{\infty} / \rho$ and noting that $u_{I}$ is non-zero only at the interface $\left(S_{I}\right)$ results in

$$
K_{0} \iint_{S_{I}(\mathbf{x}, t)} \mathbf{v} \cdot \mathbf{n d} S \approx \Phi_{\nu}-D .
$$

It is natural to define the surface averaged entrainment velocity $\left(v_{s}\right)$ as

$$
v_{S} S=\iint_{S_{I}(\mathbf{x}, t)} \mathbf{v} \cdot \mathbf{n d} S,
$$

where, $S$ is the total interface surface area, $S \equiv \iint_{S_{I}(\mathbf{x}, t)} \mathrm{d} S$. And in terms of these definitions and assuming that $D \approx 0$ in the $V$,(D2) becomes

$$
K_{0} v_{s} S \approx \Phi_{\nu}
$$

This relates the entrainment rate at the smallest scales by viscous fluxes with an average entrainment speed at the smallest scales, $v_{s}$, see also Sec. III.

Continuing in the same vein, for the RANS case (cf., Sec. IV A), we have

$$
K_{0} v_{s}^{A} S^{A} \approx \Phi_{A},
$$

where, $v_{s}^{A}$ and $S^{A}$ are defined in a manner similar to (D3) however with the RANS interface coordinate.

Similarly, for the LES case (cf., Sec. IV B):

$$
K_{0} v_{s}^{\Delta} S(\Delta) \approx \Phi_{A \Delta}+\Phi_{\nu \Delta},
$$

and similar definitions for $v_{s}^{\Delta}$ and $S(\Delta)$. And since the total entrainment should be the same at small, large, and intermediate scales, again

$$
v_{s} S \approx v_{s}^{A} S^{A} \approx v_{s}^{\Delta} S(\Delta)
$$

in accordance with (28).

${ }^{1}$ S. Corrsin and A. L. Kistler, "Free-stream boundaries of turbulent flows," NACA Technical Note No. 1244 (1955).

${ }^{2}$ O. M. Phillips, "The irrotational motion outside a free boundary layer," Proc. Camb. Phil. Soc. 51, 220 (1955).

${ }^{3}$ A. Townsend, Structure of Turbulent Shear Flow (Cambridge University Press, 1976).

${ }^{4}$ D. J. Carruthers and J. C. R. Hunt, "Momentum and scalar transport at the turbulent/non-turbulent interface of a jet," J. Fluid Mech. 165, 475-501 (1986).

${ }^{5}$ K. Sreenivasan and C. Meneveau, "The fractal facets of turbulence," J. Fluid Mech. 173, 357-386 (1986).

${ }^{6}$ J. Westerweel, C. Fukushima, J. M. Pedersen, and J. C. R. Hunt, "Momentum and scalar transport at the turbulent/nonturbulent interface of a jet," J. Fluid Mech. 631, 199-230 (2009).

${ }^{7}$ B. B. Mandelbrot, The Fractal Geometry of Nature (W. H. Freeman, New York, 1982).

${ }^{8}$ S. Lovejoy, "Area-perimeter relation for rain and cloud areas," Science 216, 185-187 (1982).

${ }^{9}$ F. Gouldin, K. Bray, and J. Chen, "Chemical closure model for fractal flamelets," Combust. Flame 77, 241 (1989).

${ }^{10}$ K. Sreenivasan, R. Ramshankar, and C. Meneveau, "Mixing, entrainment and fractal dimensions of surfaces in turbulent flows," Proc. R. Soc. London, Ser. A 421, 79-108 (1989).

${ }^{11}$ C. Meneveau and K. Sreenivasan, "Interface dimension in intermittent turbulence," Phys. Rev. A 41, 2246 (1990).

${ }^{12}$ J. Mathew and A. Basu, "Some characteristics of entrainment at a cylindrical turbulence boundary," Phys. Fluids 14, 2065-2072 (2002).

${ }^{13}$ J. Westerweel, C. Fukushima, J. M. Pedersen, and J. C. R. Hunt, "Mechanics of the turbulent-nonturbulent interface of a jet," Phys. Rev. Lett. 95, 174501 (2005).

${ }^{14}$ J. C. R. Hunt, I. Eames, C. B. da Silva, and J. Westerweel, "Interfaces and inhomogeneous turbulence," Philos. Trans. R. Soc. London, Ser. A 369, 811-832 (2011).

${ }^{15}$ M. Holzner, A. Liberzon, N. Nikitin, W. Kinzelbach, and A. Tsinober, "Small-scale aspects of flows in proximity of the turbulent/nonturbulent interface," Phys. Fluids 19, 071702 (2007).

${ }^{16}$ J. Philip and I. Marusic, "Large-scale eddies and their role in entrainment in turbulent jets and wakes," Phys. Fluids 24, 055108 (2012).

${ }^{17}$ L. S. G. Kovasznay, "Structure of the turbulent boundary layer," Phys. Fluids. 10, S25-S30 (1967).

${ }^{18}$ C. B. da Silva, R. J. N. dos Reis, and J. C. F. Pereira, "The intense vorticity structures near the turbulent/non-turbulent interface in a jet," J. Fluid. Mech. 685, 165-190 (2011).

${ }^{19}$ C. M. de Silva, J. Philip, K. Chauhan, C. Meneveau, and I. Marusic, "Multiscale geometry and scaling of the turbulent/nonturbulent interface in high Reynolds number boundary layers," Phys. Rev. Letts. 111, 044501 (2013).

${ }^{20}$ M. Khashehchi, A. Ooi, J. Soria, and I. Marusic, "Evolution of the turbulent/non-turbulent interface of an axisymmetric turbulent jet," Exp. Fluids 54, 1449 (2013).

${ }^{21}$ W. C. Reynolds, "Large-scale instabilities of turbulent wakes,” J. Fluid. Mech. 54, 481-488 (1972). 
${ }^{22}$ C. P. Chen and R. F. Blackwelder, "Large-scale motion in a turbulent boundary layer: A study using temperature contamination," J. Fluid Mech. 89, 1-31 (1978).

${ }^{23}$ C. B. da Silva and J. C. F. Pereira, "The role of coherent vortices near the turbulent/non-turbulent interface in a planar jet," Philos. Trans. R. Soc. London, Ser. A 369, 738-753 (2011).

${ }^{24}$ N. Semin, V. Golub, G. Elsinga, and J. Westerweel, "Laminar superlayer in a turbulent boundary layer," Tech. Phys. Lett. 37, 1154-1157 (2011).

${ }^{25}$ K. Chauhan, J. Philip, C. M. de Silva, N. Hutchins, and I. Marusic, "The turbulent/non-turbulent interface and entrainment in a boundary layer," J. Fluid. Mech. 740, 1-33 (2014).

${ }^{26}$ M. Holzner, A. Liberzon, M. Guala, A. Tsinober, and W. Kinzelbach, "Generalized detection of a turbulent front generated by an oscillating grid," Exp. Fluids 41, 711-719 (2006).

${ }^{27}$ C. B. da Silva and R. R. Taveira, "The thickness of the turbulent/nonturbulent interface is equal to the radius of the large vorticity structures near the edge of the shear layer," Phys. Fluids 22, 121702 (2010).

${ }^{28}$ M. Holzner and B. Lüthi, "Laminar superlayer at the turbulence boundary," Phys. Rev. Lett. 106, 134503 (2011).

${ }^{29}$ R. J. Adrian, C. D. Meinhart, and C. D. Tomkins, "Vortex organization in the outer region of the turbulent boundary layer," J. Fluid. Mech. 422, 1-54 (2000).

${ }^{30}$ C. M. de Silva, K. A. Chauhan, C. H. Atkinson, N. A. Buchmann, N. Hutchins, J. Soria, and I. Marusic, "Implementation of large scale PIV measurements for wall bounded turbulence at high Reynolds numbers," in Proceedings of the 18th Australasian Fluid Mechanics Conference, Vol. 18, edited by P. A. Brandner, and B. W. Pearce (Launceston, Australia, 2012), pp. 308-311.

${ }^{31}$ T. B. Hedley and J. F. Keffer, "Turbulent/non-turbulent decisions in an intermittent flow," J. Fluid Mech. 64, 625-644 (1974).

${ }^{32}$ R. R. Prasad and K. R. Sreenivasan, "Scalar interfaces in digital images of turbulent flows," Exp. Fluids. 7, 259-264 (1989).

${ }^{33}$ H. J. Catrakis and P. E. Dimotakis, "Mixing in turbulent jets: Scalar measures and isosurface geometry," J. Fluid. Mech. 317, 369-406 (1996).

${ }^{34}$ H. J. Catrakis, R. Aguirre, and J. Ruiz-Plancarte, "Area-volume properties of fluid interfaces in turbulence: Scale-local self-similarity and cumulative scale dependence,” J. Fluid Mech. 462, 245-254 (2002).

${ }^{35}$ N. D. Sandham, M. G. Mungal, J. E. Broadwell, and W. C. Reynolds, "Scalar entrainment in the mixing layer," in Proceedings of the Summer Program 1988 (Center for Turbulence Research, Stanford University, Stanford, CA, 1988), pp. 69-76.

${ }^{36}$ D. Bisset, J. C. R. Hunt, and M. M. Rogers, “The turbulent/non-turbulent interface bounding a far wake,” J. Fluid Mech. 451, 383-410 (2002).

${ }^{37}$ C. B. da Silva, "The behavior of subgrid-scale models near the turbulent/nonturbulent interface in jets," Phys. Fluids 21, 081702 (2009).

${ }^{38}$ J. Jimenez, S. Hoyas, M. P. Simens, and Y. Mizuno, "Turbulent boundary layers and channels at moderate Reynolds numbers," J. Fluid Mech. 657, 335-360 (2010).

${ }^{39}$ R. K. Anand, B. J. Boersma, and A. Agrawal, "Detection of turbulent/non-turbulent interface for an axisymmetric turbulent jet: Evaluation of known criteria and proposal of a new criterion," Exp. Fluids 47, 995-1007 (2009).

${ }^{40}$ G. Heckstead, "Hot-wire measurements in a plane turbulent jet," J. Appl. Mech. 32, 721-734 (1965).

${ }^{41}$ A. J. Adrian and J. Westerweel, Particle Image Velocimetry (Cambridge University Press, 2011).

${ }^{42}$ U. Piomelli, P. Moin, and J. Ferziger, "Model consistency in large eddy simulation of turbulent channel flows," Phys. Fluids 31, 1884 (1988).

${ }^{43}$ S. Pope, Turbulent Flows (Cambridge University Press, 2000).

${ }^{44}$ S. Liu, C. Meneveau, and J. Katz, "On the properties of similarity subgrid-scale models as deduced from measurements in a turbulent jet," J. Fluid Mech. 275, 83 (1994).

${ }^{45}$ B. Tao, J. Katz, and C. Meneveau, "Statistical geometry of subgrid-scale stresses determined from holographic particle image velocimetry measurements," J. Fluid Mech. 457, 35-78 (2002).

${ }^{46}$ J. Chen, C. Meneveau, and J. Katz, "Scale interactions of turbulence subjected to a straining-relaxation-destraining cycle," J. Fluid Mech. 562, 123-150 (2006).

${ }^{47}$ M. van Reeuwijk and M. Holzner, “The turbulence boundary of a temporal jet,” J. Fluid. Mech. 739, 257-275 (2013).

${ }^{48}$ P. Saarenrinne, M. Piirto, and H. Eloranta, "A correction method for measuring turbulence kinetic energy dissipation rate by PIV,” Meas. Sci. Technol. 12, 1904-1910 (2001).

${ }^{49}$ T. Tanaka and J. K. Eaton, "A correction method for measuring turbulence kinetic energy dissipation rate by PIV," Exp. Fluids 42, 893-902 (2007).

${ }^{50}$ P. Schlatter and R. Örlü, “Assessment of direct numerical simulation data of turbulent boundary layers," J. Fluid. Mech. 659, 116-126 (2010).

${ }^{51}$ From the kinetic energy balance provided by Schlatter and Örlü, ${ }^{50}$ profiles of $(1 / \rho) \partial\left(\overline{u_{3}^{\prime} p^{\prime}}\right) / \partial y$ and $1 / 2 \partial\left(\overline{u_{3} u_{i} u_{i}}\right) / \partial y$ are obtained, and integrated in the wallnormal direction with their values estimated at about $y / \delta_{99} \approx 0.9$, which is adapted to our situation.

${ }^{52}$ M. Holzner, B. Lüthi, A. Tsinober, and W. Kinzelbach, "Acceleration, pressure and related quantities in the proximity of the turbulent/nonturbulent interface," J. Fluid. Mech. 639, 153-165 (2009).

${ }^{53}$ I. Marusic and R. A. Adrian, "Eddies and scales of wall turbulence," in Ten Chapters in Turbulence, edited by P.A.Davidson, Y. Kaneda, and K.R.Sreenivasan (Cambridge University Press, 2012).

${ }^{54}$ P. Bradshaw, "Irrotational fluctuations near a turbulent boundary layer," J. Fluid. Mech. 27, 209-230 (1967). 